\title{
RECOMMENDED METHODS FOR STATISTICAL ANALYSIS OF DATA CONTAINING LESS-THAN-DETECTABLE MEASUREMENTS
}

Corwin L. Atwood Larry G. Blackwood Gerald A. Harris Cynthia A. Loehr

September 1990

Idaho National Engineering Labcratory

EG\&G Idaho, Inc. Idaho Falls, ID 83415

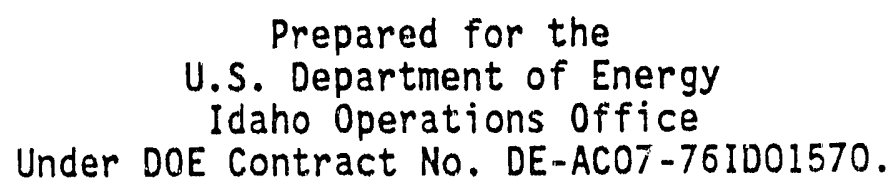




\section{ABSTRACT}

This report is a manual for statistical workers dealing with environmental measurements, when some of the measurements are not given exactly but are only reported as less than detectable. For some statistical settings with such data, many methods have been proposed in the literature, while for others few or none have been proposed. This report gives a recominended method in each of the settings considered. The body of the report gives a brief description of each recommended method. Appendix A gives example programs using the statistical package SAS, for those methods that involve nonstandard methods. Appendix $B$ presents the methods that were compared and the reasons for selecting each recommended method, and explains any fine points that might be of interest.

This is an interim version. Future revisions will complete the recommendations. 


\section{CONTENTS}

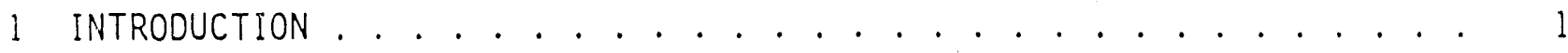

1.1 Purpose and Scope .................. 1

1.2 Report Organization .............. . . 2

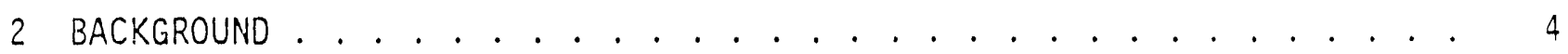

2.1 Definitions and Notations . . . . . . . . . . . . 4

2.1.2 Populations and Samples ............ . . 4

2.1.2 Moments and Percentiles ........... . . 4

2.1.3 Point Estimators and Their Properties ...... 8

2.1 .4 Interval Estimators ........... . 10

2.1 .5 Censored Data . . . . . . . . . . . . . 10

2.2 The Lognormal Distribution .............. 11

3 ONE SAMPLE . . . . . . . . . . . . . . . . . . . . . 12

3.1 Point Estimates and Confidence Intervals ......... 12

3.1.1 Maximum Likelihood Estimate (MLE) . . . . . . . 12

3.1 .2 The LR Estimate . . . . . . . . . . . . . 13

3.1.3 Recommended Method ............... 16

3.2 Tolerance and Prediction Intervals ........... 17

3.3 Goodness of fit Tests ............... 17

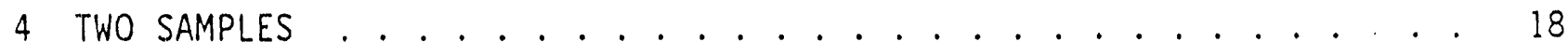

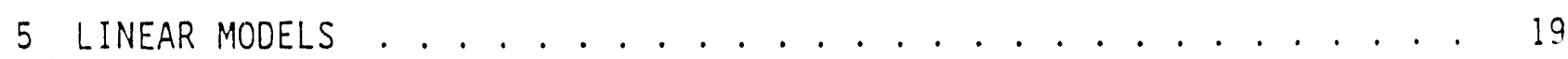

5.1 Two-Way Analysis of Variance . . . . . . . . . . 19

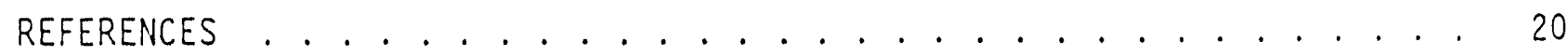

APPENDIX A. EXAMPLE PROGRAMS IN SAS ............. A-1 
APPENDIX B. TECHNICAL DETAILS AND SUPPORTING MATERIAL . . . . . . . B-1 


\section{RECOMMENDED METHODS \\ FOR STATISTICAL ANALYSIS OF DATA \\ CONTAINING LESS-THAN-DETECTABLE MEASUREMENTS}

\section{INTRODUCTION}

\subsection{Purpose ANd SCOPE}

Environmental monitoring data fraquently contain measurements that are reported as "less than detectable" (LTD). Standard textbook statistical methods do not allow such data; instead they require a11 the measurements to have known numerical values. Less well known methods have been developed in some situations to perform valid statistical analyses when some of the measurements are LTD. Many of these miethods are described in a review of the literature by Hertzler, Atwood, and harris (1989). The number of possible approaches can be bewildering. Therefore, the present report is intended to give recommended methods to use in the most commoniy encountered settings.

The body of this report considers a number of settings in which scatistical analysis is necessary, and presents the recommended method in each case. This portion of the report is written for data analysts working with environmental data. Appendix A follows the same order of topics, for each topic giving, where possible, a worked example using the statistical package SAS. Appendix $B$ is written for statisticians who are interested in the details of the possible methods. Following the same order of topics as in the body of the report, it gives the methods that were comparad and the reasons for selecting each recommended method, and explains any fine points that might be of interest. The numbering of the major sections of the appendices agrees exactly with the numbering of the body of this report, for easy cross-referencing.

The present interim version of this report contains recommendations as of 
September, 1990. Portions of the final report are not yet written. Future revisions will complete the recommendations.

\subsection{REPORT ORGANIZATION}

The next section of this report consists of background information-a review of definitions, and information on the most important probability distribution, the iognormal. Following this, the three major sections of the report consider three situations: the one-sample problem, the two-sample problem, and the 7 inear model, described briefly here.

In the one-sample setting, there is one sample of observations taken from a single population. The goal of the statistical analysis is to characterize the population in one or more ways. The parameters of interest often are:

- moments, such as the mean and variance

- percentiles, such as the median and the 95th percentile

The analysis might require

- point estimates

- confidence intervals

- tolerance intervals

- tests of hypotheses

or some other type of inference. The choice of parameters and the required analysis might be dictated by some applicable regulation.

In the two-sample setiing, there are two (independent) samples from two populations. One sample might be a control. The analysis normally involves comparing the two populations, typically by considering a difference or ratio of parameters, and computing a point estimate or interval estimate, or performing a hypothesis test. 
In the linear-model setting there are more than two samples, taken under differing conditions. The analysis includes, as special cases, analysis of variance (ANOVA) and regression. 


\section{BACKGROUND}

\subsection{Definitions and Notations}

\subsubsection{Populations and Samples}

The following definitions are stated tersely, because many of them should be familiar to readers of this report. Some quantity is being measured, such as the concentration of a contaminant. The population is the set of all possible values that could be produced, together with the distribution that determines the probabilities of the different values. The distribution is included in our definition of the population, because it is not enough to simply say that all non-negative concentrations are possible; the probabilities of the various concentrations are part of the definition of the population. For example, a smokestack could be emitting sulphur dioxide at a constant rate, and the random wind pattern causes various concentrations at a monitoring station. The population consists of all possible concentrations at the station (i.e., all nonnegative values), together with the probability distribution of these possible values (for example, some lognormal distribution.) As a second example, surface soil in a certain region could be contaminated with ${ }^{137} \mathrm{Cs}$. The population would consist of a 11 possible ${ }^{137} \mathrm{Cs}$ concentrations per 10 -gram (or other sized) unit of soil, along with the probability distribution of these concentrations.

A sample is a set of values, called observations, randomly chosen according to the population distribution. The number of observations in the sample is called the sample size, and is denoted in this report by $n$. Note, some experimenters use the term "sample" for an individual observation; in this report, a sample is always a set of numerical observations.

\subsubsection{Moments and Percentiles}

Suppose that a random quantity $x$ is generated from a orobability distribution; in other words, $x$ is a single observation to be taken from some population. We will use capital letters for random variables, and the 
corresponding lower case letters for observed values of these random variables. The use of capital $X$ in this case means that $X$ is considered random; its value has not yet been observed. The expected value of $X$, denoted $E(X)$, is the longterm average value that would be obtained if $x$ were sampled infinitely often. It is a fixed number depending on the distribution for the population, not a random variable.

A population statistic is a number that can be computed from the population distribution. A sample staistic is the analogous quantity computed from the sample observations, as if the sample were the entire population (with all the observitions having equal probability). Sample statistics are based on observed values, and are used to estimate population statistics. Two important classes of statistics are moments and percentiles, defined below.

Moments are expected values of various expressions. If $X$ denotes a single observation from a population with some distribution, the following population moments are commonly used.

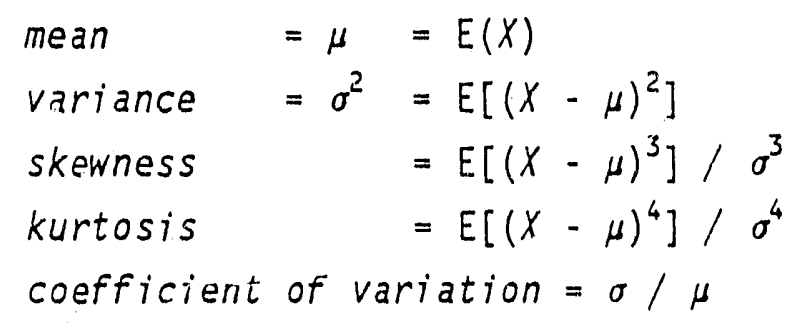

In casual use, the standard deviation, $\sigma$, is also often called a moment, even though technically it is not the expected value of anything, but is the square root of an expected value. The coefficient of variation can also be thought of as the relative standard deviation.

The skewness measures asymmetry of the distribution. A positive skewness indicates that values far from the mean are more likely on the right than on the left; a negative skewness indicates the reverse; zero skewness results from a symmetrical distribution such as the normal. Measurements of contaminants are typically positively skewed: they cannot be less than zero, and so cannot be too far to the left of the mean; however, values that are very large (relative to the mean) occasionally occur. This asymmetry yields a positive skewness. This is illustrated in Figure 2.1.1. 


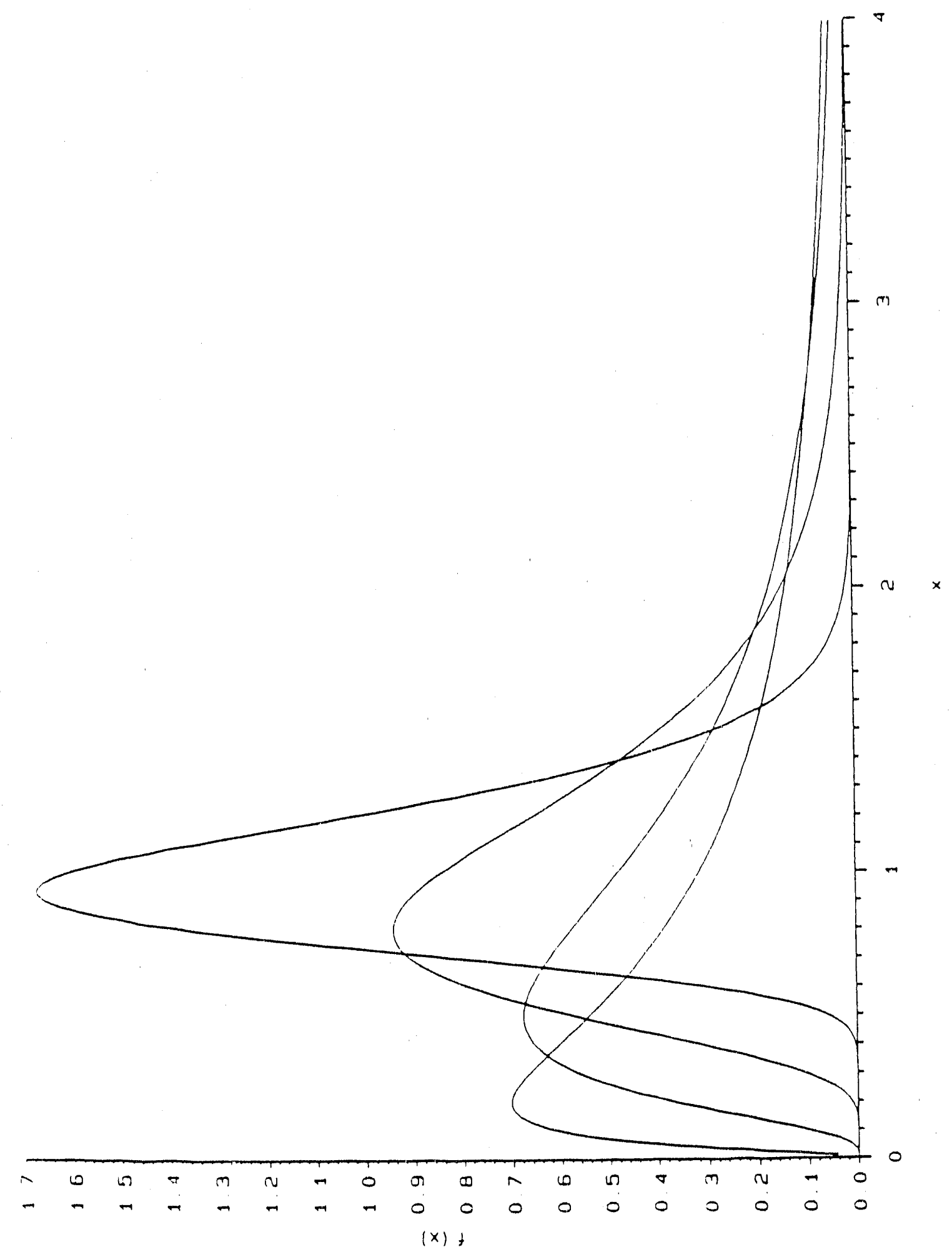

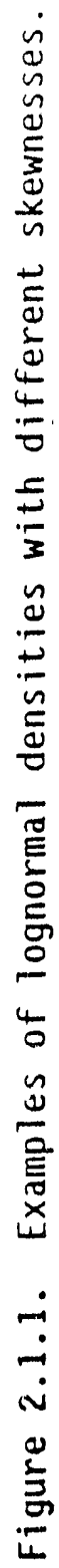


For a sample $\left(x_{1}, \ldots, x_{n}\right)$, the corresponding sample moments are

$\begin{aligned} \text { sample mean }=\bar{x} & =\Sigma x_{i} / n \\ \text { sample variance }=s^{2} & =\Sigma\left(x_{i}-\bar{x}\right)^{2} /(n-1) \\ \text { sample skewness } & =\Sigma\left(x_{i}-\bar{x}\right)^{3} / n s^{3} \\ \text { sample kurtosis } & =\Sigma\left(x_{i}-\bar{x}\right)^{4} / n s^{4}\end{aligned}$

Note that the sample variance is not the exact analogue of the population variance (with each of the $n$ observations getting weight $1 / n$ ), because the sample variance uses a divisor $n \cdot 1$ rather than $n$. This is done to make $s^{2}$ an unbiased estimator of $\sigma^{2}$. The sample moments are simple estimators of the corresponding population moments, and if $n$ is large, they are good estimators.

Percentiles differ from moments in that they are not expectations. Instead, they are values that satisfy certain probability statements. If $x$ is a random variable (that is, an observable value obeying the distribution of some population), the kth psrcentile is the number a satisfying

$$
P(X \leq a) \times 100 \%=k \% \text {. }
$$

The 50th percentile is called the median. On the average, half the observations from tine population are below the median and half are above it. The 75th percentile minus the 25 th percentile is sometimes called the interquartile range. For a lognormal distribution, the ratio

95th percentile / median

is sometimes called the error factor.

The sample percentiles are the analogues for a sample of the above population percentiles. They are often expressed in terms of the ordered observations, commonly written with subscripts enclosed in parentheses $x_{(1)} \leq x_{(2)}$ $s \ldots s x_{(n)}$. For example, if there are five observations, their values, in the order originally observed, might be 


$$
\begin{aligned}
& x_{1}=5.2 \\
& x_{2}=2.7 \\
& x_{3}=10.8 \\
& x_{4}=7.4 \\
& x_{5}=3.1
\end{aligned}
$$

The ordered observations are

$$
\begin{aligned}
& x_{(1)}=2.7 \\
& x_{(2)}=3.1 \\
& x_{(3)}=5.2 \\
& x_{(4)}=7.4 \\
& x_{(5)}=10.8
\end{aligned}
$$

The sample median in this case is $x_{(3)}$, or 5.2. In general, if $n$ is odd, the sample median is the middle ordered observation, $x_{([n+1] / 2)}$. If $n$ is even, there is no universally accepted definition of the sample median, although the average of the two middle ordered observations is commonly used. The other sample percentiles are similarly defined, with similar difficulties of detail if $n$ is not neatly divisible by the right number.

Some statistical techniques make use of ranks. The rank of an observation is an integer from 1 to $n$, with the smallest observation having rank 1 , the next smallest having rank 2, and so forth. In the above example, the rank of $x_{1}$ is 3 , the rank of $x_{2}$ is 1 , etc.

\subsubsection{Point Estimators and Their Properties}

Consider now properties of estimators. An estimator, such as a sample moment or a sample percentile, is a quantity computed from a random sample and used to approximate some unknown quantity that depends on the population. The numerical value it takes based on a particular observed sample is called an estimate. An estimator is a random variable; that is, it is based on a random sample, and if a different sample were taken the calculated estimate would be a different number. Because the estimator is a random variable, it has a mean and 
a variance. Let $\theta$ be an unknown parameter, such as $\mu$ or $\sigma$, and let $U$ be an estimator of $\theta$. For example, the sample mean $\bar{Y}$ is a natural estimator of the Dopulation mean $\mu$, the sample coefficient of variation $S / \bar{X}$ is a natural estimator of the coefficient of variation $\sigma / \mu$, atc. The value of the estimator depends on the random sample that happens to be observed. The bias of $U$, as an estimator of $\theta$, is defined as

$$
\operatorname{bias}(U)=E(U)-\theta
$$

The estimator $U$ is unbiased if the bias is zero, so that the long term average value of $U$ is the desired quantity $\theta$. In environmental contexts, the term accuracy is often used to express this idea, with high accuracy corresponding to small bias, Tie bias of an astimator cannot be considered without also considering the variance of the estimator; an unbiased estimator is of little use if its variance is enormous. In environmental contexts, precision is often used to express this idea, with high precision corresponding to small variance. The variance and bias are snmetimes combined to give an overall measure of the goodness of an estimator, the mean squared error (MSE):

$$
\text { MSE of } U=E\left[(U-\theta)^{2}\right]=\operatorname{variance}(U)+\text { bias }^{2}
$$

Often, when there are several candidate estimators of a single quantity, the analyst will select the estimator with the smallest MSE. This method may not give the best estimate for the particular data set being analyzed (if we knew which estimate was best, we would not need to estimate anything), but on the averaje the meihod has the smallest squared error.

One more desilable property of an estimator is robustness. The properties of an estimator, such as it.s variance or bias, are usually derived based on some assumptions, for example the assumption that the population is lognormal. An estimator is robust if its properties do not change greatly when the underlying assumptions are only approximately true. Robustness studies are often empirical; for example, an estinator derived under the lognormal assumption can be studied using simulated data sets from other skewed populations such as gamma or Weibull. 


\subsubsection{Interval Estimators}

An unknown parameter such as $\mu$ or $\sigma$ can be estimated by an interval as well as by a point estimate. The interval is intended to include the value of the parameter with high probability. Such an interval is called a confidence interval. It is computed from the sample; therefore different random samples would produce different intervals. For a $k \%$ confidence interval, the probability that the interval contains the unknown parameter is $k \%$. This confidence level, $k \%$, is a property of the method, not of the particular calculated interval. We never know whether we got an unlucky sample, so that the interval misses the unknown value of the parameter, or a more typical sample, so that the interval contains the parameter. We only know that if we took many samples and computed the many resulting confidence intervals, $k \%$ of the intervals would contain the true value of the parameter.

There are other kinds of intervals, tolerance intervals and prediction intervals. Their definitions will be added to a future revision, when the recommiended methods are given.

\subsubsection{Censored Data}

Data are censored if some or all of the observation values are not known exactly. For a censored observation, it is only known that the value is bounded by some number, or is in some interval. In environmental contexts, some observations are reported as less than detectable (LTD); this means that the value is less than some known detection 1 imit (denoted $D L$ ), but is not known more exactiy.

In the literature on censored data, two types of censoring are distinguished. In Type I censoring, an observation is censored if it is beyond a predetermined value. This is the type of censoring that is relevant in the environmental setting, in which observations are reported as less than detectable if they are below a fixed value DL. Note, the value of the detection limit does not have to be the same for all the observations, but it must exist in principle and be independent of the values of the observed data. By contrast, in Type II 
censoring the number of censored observations is some predetermined fraction of the sample size, and the cutoff value between the censored and uncensored observations is random. This kind of censoring occurc in survival tests, when it is decided in advance to watc'i a number of items until a certain fraction of them have failed. Type II censoring is not used in the context of this report.

\subsection{THE LOGNORMAL DISTRIBUTION}

Environmental measurements considered here are typically measurements of concentrations, measured in parts per million or picocuries per liter or similar units. The concentration being measured varies randomly, depending on the particular sample of air, soil, water, or other medium, and it cannot be negative.

The most important distribution used to model such a random variable is the lognormal distribution. The defining relation is that $X$ is lognormal if its logarithm is normal. By custom, the natural logarithm $\ln (X)$ is used. The lognormal distributic, is so widely used because of its simple relation to the normal distribution. If $x_{1}, \ldots, x_{n}$ are a set of measurements, define $y_{;}=$ $\ln \left(x_{i}\right)$. Assuming that the $x^{\prime} s$ come from a lognormal population means that the $y^{\prime} s$ come from a normal population. Therefore many classical methods can be used with the $y^{\prime} s$, and the results translated back to give results about the population of the $x^{\prime} s$. For an introductory discussion of the lognormal distribution, see the relevant section of Appendix $B$ and the references mentioned there.

Many of the statistical methods presented here are based on lognormality. The assumption of lognormality is usually made for convenience, not for any theoretical reason. To be recommended, therefore, a method must be robust, that is, it must perform well even when the assumption of lognormality is not exactly correct. 


\section{ONE SAMPLE}

\subsection{Point Estimates and Confidence Intervals}

Two types of estimation are described, yielding the maximum likelihood estimate (MLE) and the LR estimate of Gilliom and Helse? (1986). The MLE is best for estimating percentiles, such as the median or the goin percentile of the distribution, according to extensive simulations performed by Gilliom and Helsel. (Gilliom and Helsel do not consider sample percentiles as estimates.) For estimating moments of the distribution, such as the mean and variance, the MLE is as good as LR when the distribution is not too highly skewed. LR is best for estimating the moments when the distribution is very skewed. We recommend using the MLE. If the distribution seems extremely skewed and moments are being estimated, compute the LR fatimate as wel1. Suppose that the two methods give markedly different estimates. Then, unless regulations require it, do not use either estimate of the moments-do not estimate moments at all, but estimate percentiles instead. These points are explained in more detail in the sections below.

\subsubsection{Maximum Likelihood Estimate (MLE)}

When the data are censored, the MLE must be computed using numerical iteration-there is no simple formula. Maximum likelihood estimation for censored data is therefore most easily implemented with survival analysis programs in commercial packages. These progran; generally allow a number of distributional assumptions. For example, the LIFEREG procedure in SAS allows the Weibull, exponential, gamma, normal, lognormal, logistic, and loglogistic distributions. A distribution must be assumed before the MLE can be computed. Gilliom and Helsel (1986) assume the lognormal distribution, and show that the MLE estimates percentiles as wel? as other estimators do, even if the data come from some distribution other than the assumed lognormal. The MLE also estimates moments well when the distribution is not too skewed, but is very biased for estimating the moments when the distribution is strongly skewed. 
See Appendix A for an example calculation of the MLE of the lognormal parameters using LIFEREG. The MLEs of moments or percentiles are obtained by substituting the parameter estimates into the appropriate formulas.

Approximate confidence intervals are obtained by using the asymptotically normal distribution for the MLE: The SAS procedure LIFEREG gives confidence intervals for the percentiles, and a SAS program given in Appendix $A$ gives approximate confidence intervals for the mean and variance of the distribution.

\subsubsection{The LR Estimate}

The term LR stands for "lognormal regression." The LR estimate is based on the assumption that the observations are lognormally distrihuted, but it seems to be robust, that is, it seems to work well even if the observations in fact follow a somewhat different distribution. The idea is simple: plot the uncensored values on ?ognormal probability paper (or equivalently, plot their logs on normal probability paper). The points should fall approximately on a straight line. Fit a straight line to these points, and extrapolate the line back below the detection limit to estimate values for the censored observations. Use these calculated values, together with the observed uncensored values, to calculate the sample mean and sample variance.

To illustrate the method, suppose that the ordered observations are the following:

$\begin{array}{ccc}\text { number } & \text { observation } & \frac{\ln \text { (observation) }}{1} \\ 2 & <4.1 & - \\ 3 & <4.5 & - \\ 4 & 4.5 & 1.50 \\ 5 & 5.3 & 1.67 \\ 6 & 5.3 & 1.67 \\ 7 & 5.6 & 1.72 \\ 8 & 7.7 & 2.04 \\ 9 & 8.5 & 2.14 \\ & 9.5 & 2.25\end{array}$




$\begin{array}{rrr}10 & 9.6 & 2.26 \\ 11 & 11.0 & 2.40 \\ 12 & 17.0 & 2.83 \\ 13 & 17.0 & 2.83\end{array}$

This data set is also used in Appendix B. There are 13 observations, of which two are less than detectable. On normal probability paper, plot the logs of the uncensored observations against the points $3 / 14,4 / 14, \ldots, 13 / 14$. (The number 14 is the number of observations plus 1 , and the numerators go from 3 to 13 because the ordered observations 3 through 13 are uncensored.) This is shown graphically in Figure 3.1.1. Fit a straight line through the points, and extrapolate them backward to estimate the censored values, shown by open circles on the line in the figure. Convert these replacement log-values back to the original units and use the resulting data to calculate the sample mean and sample variance.

There is one detail that must be made more exact, in order to actually carry out the above procedure: how, precisely, should the fitlad line be determined? The formulas for least-squares fitting, rather than the above eyeball fitting, are given in Appendix B. An example using SAS is given in Appendix A.

It is possible that a calculated $x$, based on extrapolating back using the LR method, may be greater than the detection limit, even though the calculated value is supposed to be used in place of a measurement that was less than detectable. This is a warning indication, perhaps suggesting that the ciata do not fit the assumed model very well or that the distribution is highly skewed. In this case, consult a statistician.

To get confidence int zrvals, Helsel and Gilliom (1986) give a method. We do not describe it here, because we recommend use of the LR method only as a check on the adequacy of the MLE. As a simple check, if the LR estimate of the mean or variance is outside the $\mathrm{ML} 90 \%$ or $95 \%$ confidence interval ror that parameter, then the two estimates are inconsistent. 


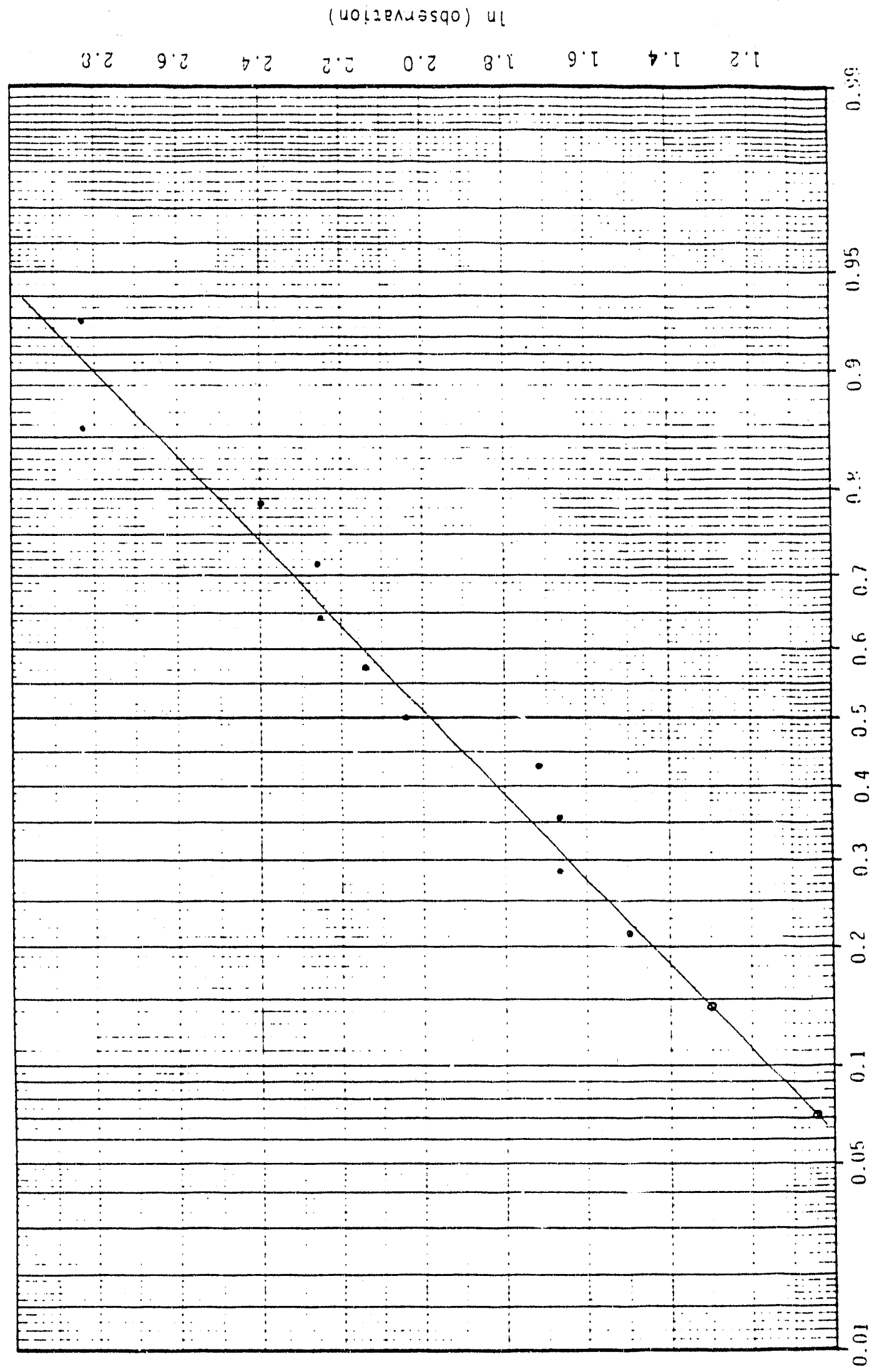

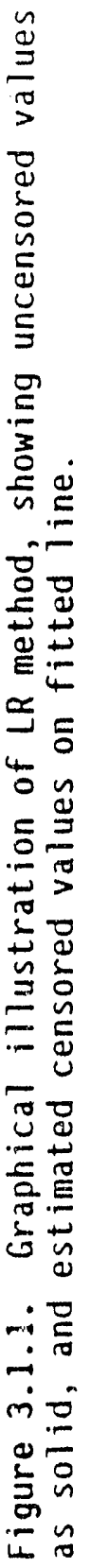




\subsubsection{Recommended Method}

Use the MLE. Trouble can arise if the distribution is very skewed. possible signs of this are:

- A large estimated coefticient of variation

(Tentative recommendation: if the coefficient of variation is as large as 1.0, then the skewness is quite high and the variance should not be estimated. This is based on uncensored data. Perhaps a smaller cutoff than 1.0 should be used.)

- Inconsistency between the LR and ML estimates of the moments

- The presence of many censored observations, plus some very large observations, without many intermediate observations

In this case, consult a statistician. If possible, take more observations. Try to avoid using estimates of the moments (mean and variance), which are strongly influenced by extreme observations; instead estimate percentiles, which are less sensitive to the values of extreme observations.

The moments of an extremely skewed distribution are not very practical descriptors of the distribution. When the underlying data distribution has heavy tails, the probability of an extreme occurrence is great enough to severely inflate the moments of the distribution. Yet a researcher might reasonably expect to do a lifetime of work without encountering one of these rare occurrences. Therefore, when dealing with highly skewed distributions, it is best to avoid using moments for characterizing the distribution. Even if the mean must be used, for example because an average exposure is of regulatory interest, try to avoid estimating the variance of the distribution; the variance is more heavily influenced by rare extreme values than the mean is.

A future revision to this report will consider sample percentiles, such as the sample median, as estimators of the population percentiles. This may alter the above recommendations. 


\subsection{Tolerance and Prediction Intervals}

This will be added in a future revision.

\subsection{Goodness of Fit Tests}

This will be added in a future revision. 


\section{TWO SAMPLES}

It is a common practice in environmental data analysis to compare two groups. For example, one may wish to compare site concentrations of a contaminant to background concentrations. In ground water monitoring, one often compares downgradient concentrations to upgradient concentrations to assess whether i contaminant has entered the ground water. As always, some of the data may be censored. In this situation, the Wilcoxon rank sum test (al so called the Mann-Whitney test) is recommended. The technique is easy to use and widely available on commercial statistical software.

When there is a single detection limit (DL), the test is done as follows. First replace all the LTD values by a value no larger than DL. It does not matter whether this value is $\mathrm{DL}$ or $\mathrm{DL} / 2$ or 0 or some other small value; the resulting ranks will be the same. Now rank all the data from the two groups together, assigning the average rank to any tied data. Then for each of the two groups compute the sum of those ranks, and compare the two sums. When there is a large difference between the sums of the ranks, there is strong evidence of a difference between the groups. This use of ranks makes the test robust to the form of the distribution.

When there are multiple detection limits, the modifications are more complex, but are presented by Millard and Deveral (1988). They will be added to a future version of this report. 


\section{LINEAR MODELS}

The methods of this section assume that the original data are lognormal. Therefore taking natural logarithms of the observations transforms them to a sample from a normal population. Standard 1 inear-model methods may be used on normal data, to perform inferences about the means under the assumption of a constant variance. Therefore, the results translate to inferences about the medians of the original lognormal data, under the assumption of a constant coefficient of variation.

\subsection{TWo-Way Analysis of Variance}

The following recommendation in this case is based on studies of a $2 \times 2$ table, with either 3 or 8 observations per ce11, and up to $60 \%$ of the observations censored, as described in Section B-5.1. The recommended method is to replace each censored observation by half the detection limit, to take the natural logarithm each observation, and to perform the usual analysis of variance on these transformed observations. 


\section{REFERENCES}

Robert J. Gilliom and Dennis R. Helsel, "Estimation of Uistributional Parameters for Censored Trace Level Water Quality Data: 1. Estimation Techniques, "Water Resources Research, 22, 1986, pp. 135-146.

Dennis R. Helsel and Robert J. Gilliom, "Estimation of Distributional Parameters for Censored Trace Level Water Quality Data: 2. Verification and Apolications," Water Resources Research, 22, 1986, pp. 147-155.

C. L. Hertzler, C. L. Atwood, and G. A. Harris, Current Methods of Handling Lessthan-Detectable Measurements and Detection Limits in Statistical Analysis of Environmental Data, EGG-SARE-8609, September 1989.

S. P. Millard and S. J. Deveral, "Nonparametric Statistical Methods for Comparing Two Sites Based on Data with Multiple Nondetect Limits," Water Resources Research, 24, 12, 1988; pp. 2087-2098. 


\section{APPENDIX A \\ EXAMPLE PROGRAMS IN SAS}

A- $1 / 2$ 


\section{CONTENTS}

A-1 INTRODUCTION . . . . . . . . . . . . . . . . . . . . A-5

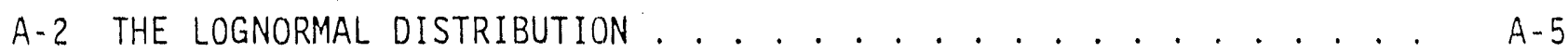

A-3 ONE SAMPle... . . . . . . . . . . . . . . . . . . . A-5

A-3.1 Point Estimates and Confidence Intervâls. . . . . . . . A-5

A-3.1.1 Maximum Likelihood Estimation . . . . . . . . . A-5

A-3.1.2 LR Estimates................ A-9

A-4 TWO SAMPLES ................... A-13

A-5 LINeAR MODEL . . . . . . . . . . . . . . . . A-13

A-5.1 Two-Way Analysis of Variance............. A-13

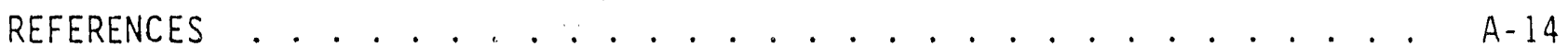




\section{APPENDIX A \\ EXAMPLE PROGRAMS IN SAS}

\section{A-1 INTRODUCTION}

This appendix gives example SAS programs (SÄS, 1988a,b,c) for performing some of the statistical techniques used in this report. To run one of the examples, copy the program printed here to a file in your SAS directory, with extension 'sas'. For example, suppose you name it 'myprog.sas'. Then you may run it by entering the DOS command 'sas myprog'. The results will appear in files named 'myprog. $\log ^{\prime}$ and 'myprog.1st'. To adapt the program to use your own data rather than example data, change the data lines in the example.

If no special programming is necessary in order to carry out the method in SAS, then no example is given here. Examples are given only when a nontrivial sequence of steps is needed. Therefore, nonparametric methods and methods for which censored values are replaced by an easily calculated number are not illustrated here.

\section{A-2 THE LOGNORMAL DISTRIBUTION}

This section is included to keep the numbering system parallel to that in the body of the report. The examples in this appendix either assume that the observations are lognormal, or that they have already been transformed by taking natural logarithms and are therefore normal.

\section{A-3 ONE SAMPLE}

\section{A-3.1 Point Estimates and Confidence Intervals}

\section{A-3.1.1 Maximum Likelihood Estimation}

The 801 lowing SAS program uses the SAS procedure LIFEREG to obta in maximum 
likelihood estimates (MLES), assuming a lognormal population with mean $\alpha$ and standard deviation $\beta$. The corresponding normal population has mean and standard deviation $\mu$ and $\sigma$, respectively. MLEs are found for $\mu, \sigma, \alpha, \beta^{2}$, and for selected percentiles of the lognormal population. The asymptotic variances are found for the estimators, so that approximate confidence intervals can be calculated. The procedure can easily be modified so that a distribution other than the lognormal is used; in this case the parameters called INTERCPT and SCALE by PROC LIFEREG no longer correspond to a normal $\mu$ and $\sigma$, but the other results are unchanged.

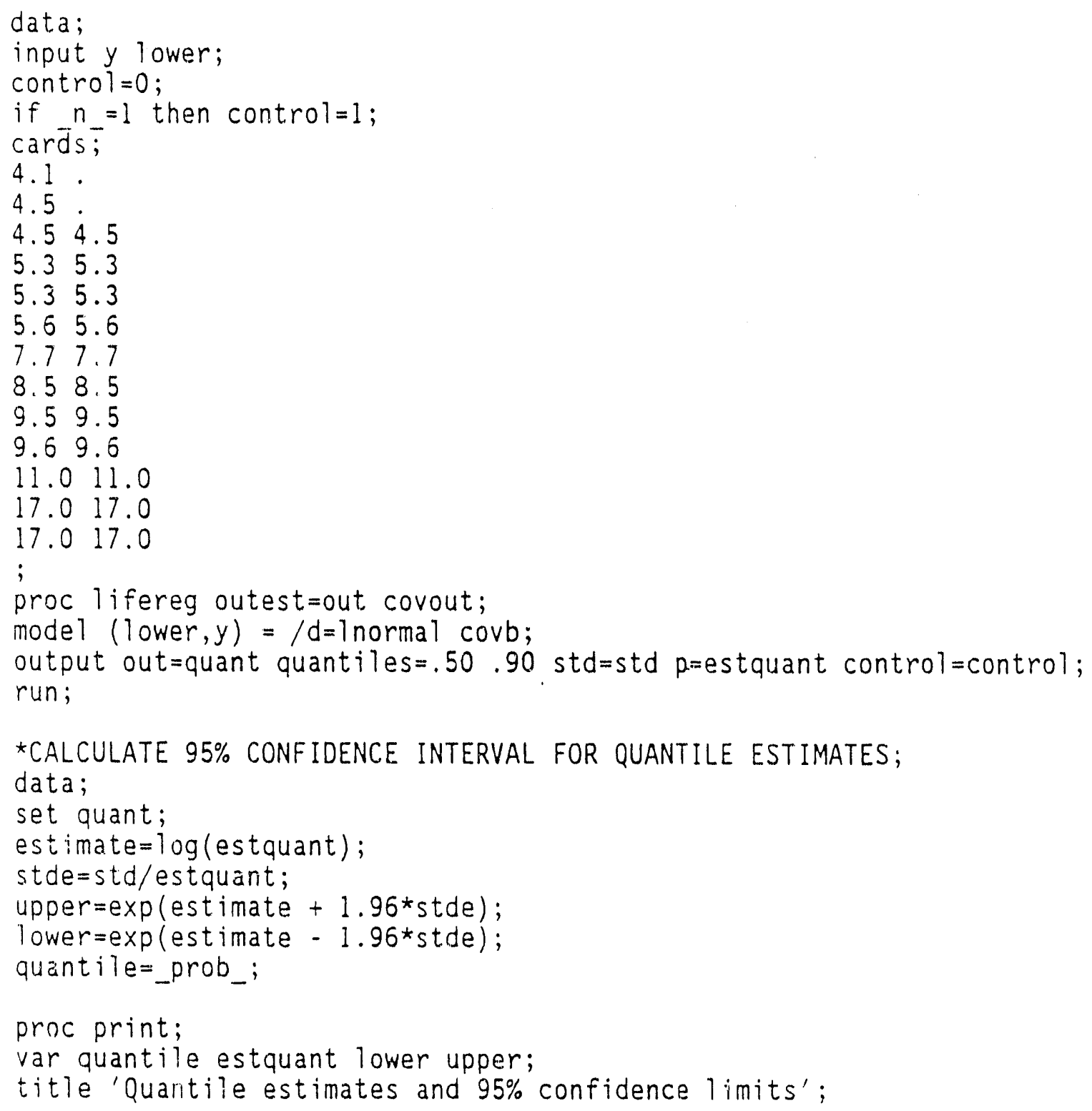


*PUT PARAMETER ESTIMATES AND COV MATRIX INTO MACRO VARIABLES;

data one;

set out;

if type ='PARMS' then do;

calT symput (' $M$ ', intercep);

call symput ('S',_scale_);

end;

if name $=$ 'INTERCPT' then do;

calT symput (' $A$ ', intercep);

call symput ('C',_scale_);

end;

if _name_='SCALE' then call symput ('B',_scale_);

*RETRIEVE MACRO VARIABLES, CALCULATE LOGNORMAL MEAN AND VARIANCE AND; *COVARIANCE MATRIX;

data one;

*MU $=$ NORMAL MEAN;

$m u=$ symget $(' M$ ') ;

*SIG=NORMAL STANDARD DEV.;

sig=symget $\left({ }^{\prime} S^{\prime}\right)$;

*KII=VARIANCE OF MU;

$k 11=$ symget $(' A$ ');

$\star K 22=V A R I A N C E$ OF SIG;

$k 22=$ symget $\left({ }^{\prime} B^{\prime}\right)$;

*K12=COVARIANCE OF MU AND SIG;

$k 12=\operatorname{symget}\left({ }^{\prime} C^{\prime}\right)$;

* INVERT AND TAKE NEGATIVE OF $K$ MATRIX TO GET INFO MATRIX J;

det $=k 11 * k 22-k 12 * * 2$;

$j 11=-k 22 /$ det

$j 22=-k 11 /$ det;

$j 12=k 12 /$ det

* CAlCulate logNoRmal mean and variance;

*A=LOGNORMAL MEAN;

$a=\exp (m u+.5 *$ sig**2);

$\star B 2=$ LOGNORMAL VARIANCE;

b2 $=\exp \left(2 * m u+\operatorname{sig}{ }^{\star \star 2} 2\right) *(\exp (\operatorname{sig} \star \star 2)-1) ;$

*CALCULATE INFORMATION MATRIX FOR lOGNORMAL MEAN AND VARIANCE;

*DUDA $=$ DERIVATIVE OF MU WRT A, DSDB2=DERIVATIVE OF SIG WRT B2, ETC.;

$\operatorname{duda}=(1 / a) *(1+1 /(1+a * * 2 / b 2))$;

dudb $2=-1 /(2 *(b 2+a * \star 2))$;

$d s d a=-1 /\left(\operatorname{sig} a^{\star}(1+a * \star 2 / b 2)\right)$;

$d s d b 2=1 /(2 * \operatorname{sig} *(b 2+a * \star 2)) ;$

$k 11=j 11 *$ duda**2+2*j12*dsda*duda $+j 22 * d s d a * * 2$;

$k 22=j 11 * d u d b 2 * \star 2+2 * j 12 * d s d b 2 * d u d b 2+j 22 * d s d b 2 * \star 2 ;$

$k 12=j 11 * d u d a * d u d b 2+j 12 *(d s d a * d u d b 2+d s d b 2 * d u d a)+j 22 \star d s d a * d s d b 2$; 
*INVERT AND TAKE NEGATIVE OF NEW K MATRIX TO GET VAR/COV MATRIX J; det $=k 11 * k 22-k 12 * \star 2$;

$v a=-k 22 /$ det

$v b 2=-k 11 /$ det;

$c a b 2=k 12 /$ det;

file print;

title 'Estimates of lognormal mean and variance';

put;

put 'Lognormal mean, $a=' a$;

put;

put 'Lognormal variance, b2 $=$ ' b2;

put;

put 'Asymptotic variance of estimator of $a={ }^{\prime} \mathrm{va}$;

put;

put 'Asymptotic variance of estimator of b2 $=$ ' vb2;

put;

put 'Asymptotic covariance of estimators of $a$ and b2 $=$ ' cab2;

run;

The output from running the above example is printed here, copied from the DOS file with extension '1st'. In the output of PROC LIFEREG, the variables called INTERCPT and SCALE are the normal parameters $\mu$ and $\sigma$, respectively.

SAS 10:51 Thursday, September 27, 1990

LIFEREG PROCEDURE

Data Set $=$ WORK $\cdot$ DATA

Dependent Variabie $=\log ($ LOWER

Dependent Variable $=\log (Y)$

Noncenscred Values $=\quad 11$ Right Censored Values $=\quad 0$

Left Censored Values $=2$ Interval Censored Values $=0$

Log Likelihood for LNORMAL - 10.86329568

SAS 10:51 Thursday, September 27, 1990

LIFEREG PROCEDURE

Variable DF Estimate Std Err ChiSquare Pr>Chi Label/Value

INTERCPT $\quad 11.974307890 .151434 \quad 169.9744 \quad 0.0001$ Intercept

SCALE I $0.53518773 \quad 0.118413$

Normal scale parameter 


\begin{tabular}{lrr}
\multicolumn{3}{c}{ Estimated Covariance Matrix } \\
\\
INTERCPT & SCALE \\
INTERCPT & 0.022932 & -0.001621 \\
SCALE & -0.001621 & 0.014022
\end{tabular}

Quantile estimates and $95 \%$ confidence limits 3 10:51 Thursday, September 27, 1990

OBS QUANTILE ESTQUANT LOWER UPPER

$\begin{array}{rrrrr}1 & 0.5 & 7.2016 & 5.35214 & 9.6902 \\ 2 & 0.9 & 14.2988 & 9.57762 & 21.3474\end{array}$

Estimates of lognormal mean and variance

4

10:51 Thursday, September 27, 1990

Lognorma 1 mean, $a=8.310 \$ 0888$

Lognormal variance, b2 $=22.905916033$

Asymptotic variance of estimator of $a=1.7413221958$

Asymptotic variance of estimator of b2 $=241.86162368$

Asymptotic covariance of estimators of $a$ and b2 $=14.411935268$

\section{A-3.1.2 LR Estimates}

The following SAS program carries out the LR method for estimating the mean and variance of a population. The method is based on the assumption that the population is lognormal.

*INPUT DATA, CENS $=1$ IF MEASUREMENT CENSORED (I.E. Y IS A DETECTION LIMIT);

$\star$ CENS $=0$ OTHERWISE;

data one;

input y cens;

cards;

4.11

4.51

4.50

5.30 
5.30

5.60

7.70

8.50

9.50

9.60

11.00

17.00

17.00

*SORT DATA SO THAT N_ CONTAINS RANKS;

proc sort;

by $y$;

data one;

set one;

rank $=N_{-}$;

*CALCULATE LOGS OF UNCENSOREd VAlUes;

$z=\log (y)$;

if cens $=1$ then $z={ }^{\prime} .^{\prime}$;

*CALCULATE NORMAL SCORES FCR RANKS;

proc rank out=out normal=vW; var rank; rarks nscore;

*PERFORM REGRESSION, OUTPUT PREDICTED VALUES;

data one;

set out;

proc reg;

model $z=n s c o r e$

output out=out predicted=pred;

*CALCULATE MOMENTS USING PREDICTED VALUES FOR CENSORED DATA;

data one;

set out;

if cens $=0$ then $y=\exp$ (pred);

proc univariate;

var $y$;

run ;

The results of running the above program are printed here, copied from the DOS file with extension '1st'. The analysis-of-variance table is prodliced from the regression of the observations on the normal scores. The fact that R-square is large indicates that the fit is good. For a more careful check of this, residual plots should be examined. These are obtained by running PROC REG with 
option R; that is, the model line is

model $z=n s c o r e / r$;

After the censored observations have been replaced based on extrapolating the regressior iine to the censored obs rvations, the sample is described by PROC LNIVARIATE. The sample moments are given in the table of moments below. The sample mean is 8.39602 and the sample variance is 18.5196 . These are quite close to the MLEs for the mean and variance found in Section $4-3.1 .1$ using the same data. This indicates that the MLE is probably acceptable.

SAS 11:16 Wednesday, September 26, 1990

Mode 1: MODEL1

Defendent Variable: $z$

Analysis of Variance

$\begin{array}{lrrrrr}\text { Source } & \text { DF } & \begin{array}{r}\text { Sum of } \\ \text { Squares }\end{array} & \begin{array}{r}\text { Mean } \\ \text { Square }\end{array} & \text { F Value } & \text { Prob }>F \\ \text { Model } & 1 & 1.99825 & 1.99825 & 207.762 & 0.0001 \\ \text { Error } & 9 & 0.08656 & 0.00962 & & \\ \text { C Total } & 10 & 2.08481 & & & \end{array}$

Root MSE

$\begin{array}{lll}0.09807 & R-s q u a r e & 0.9585 \\ 2.12008 & \text { Adj R-sq } & 0.9539 \\ 4.62582 & & \end{array}$

C.V.

4.62582

SAS 11:16 Wednesday, September 26, 1990

Parameter Estimates

$\begin{array}{lrrrrr}\text { Variable } & \text { DF } & \begin{array}{r}\text { Parameter } \\ \text { Estimate }\end{array} & \begin{array}{r}\text { Standard } \\ \text { Error }\end{array} & \begin{array}{r}\text { T for } \mathrm{HO} \text { Parameter }=0 \\ \text { Parob }>|T|\end{array} & \text { Prob } \\ \text { INTERCEP } & 1 & 1.973496 & 0.03126956 & 63.112 & 0.0001 \\ \text { NSCORE } & 1 & 0.636532 & 0.04416777 & 14.414 & 0.0001\end{array}$


UNIVARIATE PROCEDURE

$\operatorname{Variable}=Y$

\section{Moments}

\begin{tabular}{|c|c|c|c|}
\hline $\begin{array}{l}N \\
\text { Mern } \\
\text { Std Dev } \\
\text { Skewness } \\
\text { USS } \\
\text { CV } \\
\text { T:Mean=0 } \\
\text { Sgn Rank } \\
\text { Num }{ }^{\wedge}=0\end{array}$ & $\begin{array}{r}13 \\
8.39602 \\
4.303441 \\
1.164159 \\
1138.646 \\
51.25572 \\
7.034436 \\
45.5 \\
13\end{array}$ & $\begin{array}{l}\text { Sum Wgts } \\
\text { Sum } \\
\text { Variance } \\
\text { Kurtosis } \\
\text { CSS } \\
\text { Std Mean } \\
\text { Prob }>\mid T \\
\text { Prob }>\mid \text { S }\end{array}$ & $\begin{array}{r}13 \\
109.1483 \\
18.5196 \\
0.864622 \\
222.2352 \\
1.19356 \\
0.0001 \\
0.0002\end{array}$ \\
\hline
\end{tabular}

SAS 11:16 Wednesday, September 26, 1990 UNIVARIATE PROCEDURE

Variable $=Y$

\begin{tabular}{crrr}
\multicolumn{5}{c}{ Quantiles (Def=5) } \\
$100 \%$ Max & 18.28921 & $99 \%$ & 18.28921 \\
$75 \%$ Q3 & 10.31704 & $95 \%$ & 18.28921 \\
$50 \%$ Med & 7.195789 & $90 \%$ & 14.19864 \\
$25 \%$ Q1 & 5.018819 & $10 \%$ & 4.347119 \\
$0 \%$ Min & 4.1 & $5 \%$ & 4.1 \\
Range & 14.18921 & $1 \%$ & 4.1 \\
Q3-Q1 & 5.298225 & & \\
Mode & 4.1 & &
\end{tabular}




\section{UNIVARIATE PROCEDURE}

Variable $=Y$

\section{Extremes}

\begin{tabular}{|c|c|c|}
\hline $\begin{array}{r}\text { Lowest } \\
4.1( \\
.347119 \\
4.5( \\
.018819( \\
.699742(\end{array}$ & Obs & $\left\{\begin{array}{r}\text { Highest } \\
9.084513 \\
10.31704 \\
11.9112 \\
14.19864 \\
18.28921\end{array}\right.$ \\
\hline
\end{tabular}

\section{A-4 TWO SAMPLES}

When there is only one detection limit, the Wilcoxon-Mann-Whitney test can be performed in SAS by using the WILCOXON option in PROC NPARIWAY. For this, assign the value 0 (or the detection limit) to all observations that are all less than the detection limit; SAS then treats them as ties. When there are multiple detection limits, the method is more complex, and will be discussed in a future revision of this report.

The method of iterative least squarıs (ILS) has not been programmed in SAS, because to do so would be quite difficult. Other languages, such as fortran or Pascal, are more suited to tasks such as ILS.

\section{A-5 LINEAR MODEL}

\section{A-5.1 TWO-Way ANalysis of Variance}

To implement the recommended method, replace al1 censored observations by half the detection limit. Take natural logarithms of the resulting set of values (uncensored observations plus replaced censored values), and perform a two-way analysis of variance using PROC ANOVA. 


\section{REFERENCES}

SAS Institute Inc., SAS Language Guide for Personal Computers, Release 6.03 Edition, Cary, NC: SAS Institute Inc., 1988a.

SAS Institute Inc., SAS Procedures Guide, Release 6.03 Edition, Cary, NC: SAS Institute Inc., 1988b.

SAS Institute Inc., SAS/STAT User's Guide, Release 6.03 Edition, Car'j, NC: SAS Institute Inc., 1988c. 


\section{APPENDIX B}

TECHNICAL DETAILS AND SUPPORTING MATERIAL 


\section{CONTENTS}

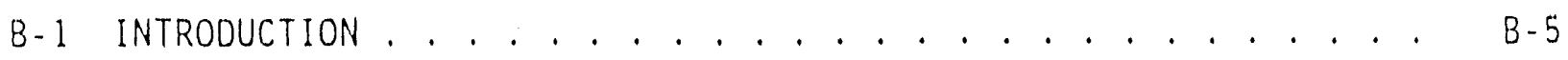

B-2 THE LOGNORMAL DISTRIBUTION ............ B-6

B-2.1 Introduction . . . . . . . . . . . . B B 6

B-2.2 Justification for Using the Lognormal . . . . . B-6

B-2.3 Properties of Lognormal Variables and Their Implications for Statistical Analysis ............. B-8

B-2.3.1 Mathematical Properties . . . . . . . . B-8

B-2.3.2 Measures of Central Tendency ........ B-14

B-2.3.3 Measures of Dispersion ......... B-17

B-2.3.4 Confidence Intervals and Hypothesis Tests ... B-17

B-2.4 Discussion and Recommendations ......... B-19

B-2.4.1 Use of the Logarithmic Transformation .... B-19

B-2.4.2 Alternatives to the Lognormal Distribution... B-19

B-2.4.3 Robustness of Lognormal Analysis Methods .... B-20

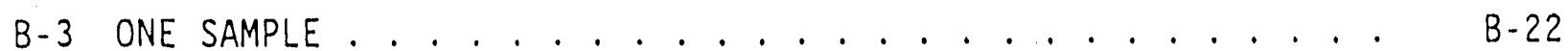

B-3.1 Point Estimates and Confidence Intervals ...... B-22

B-3.1.1 Maximum Likel ihood Methods ......... B-22

B-3.1.2 The LR Method ............ B-23

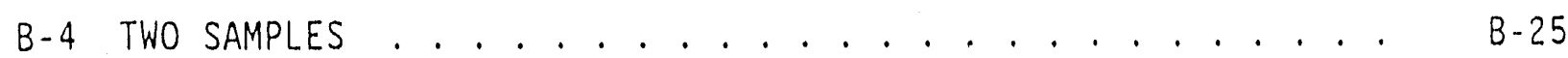

B-4.1 Two-Sample Testing: Nonparametric Methods ..... B- B-25

B-4.1.1 Simulation Methods ........... B-26

B-4.1.2 Discussion ............. B-26

B-4.2 Two-Sample Testing: Iterative Least Squares . . . . B-29

B-4.2.1 Specification of the Method ........ B-29

B-4.2.2 Simulations and Competing Methods ..... B-33

B-4.2.3 Results ............. B-35 
B-5 LINEAR MODELS . . . . . . . . . . . . . . . . . . B-39

B-5.1 Simulations on Two-Way Andiysts of Variance with Replacements for Censured Values .......... . B-39

B-5.1.1 Introduction.............. B-39

B-5.1.2 Simulation Technique........... B-40

B-5.1.3 Simulation Results............. B B-47

B-5.1.4 Conclusions ............... . B-54

B-5.1.5 Programs for Data Generation and ANOVA Simulation B.59

REFERENCES ...................... B-67 


\section{APPENDIX B TECHNICAL DETAILS AND SUPPORTING MATERIAL}

\section{$B-1$ INTRODUCTION}

This appendix contains fuller details regarding the methods recommended, as well as mention of methods that were considered but not ultimately recommended. The comparisons that form the basis of the recommendations are described. This appendix is written at a more demanding technical level than the body of the report. It is intended for statisticians who need to know the details. 


\section{B-2 THE LOGNORMAL DISTRIBUTION}

\section{B-2.1 INTRODUCTION}

This section is adapted from Blackwood (1990). For a more complete discussion, refer to that report.

A random variable is said to be lognormally distributed if its natural logarithm follows a normal distribution. Lognormal distributions allow only positive, nonzero values and are positively skewed. Since radiological and other measuremer...; in the environment are often intrinsically positive as well as highly skewed, the assumption of a lognormal distribution for these variables is common.

Much work already exists summarizing the properties and applications of the lognormal distribution. Most notable are the books by Aitchison and Brown (1957) and Crow and Shimizu (1988). However, these treatments concentrate more on the theoretical aspects of the distribution, or on non-environmental applications. The purpose of this section is to review the properties of the lognormal distribution in the context of the arialysis of environmental data, emphasizing the issues related to data analysis and interpretation. The focus is on how the "usual" approach to estimation and hypothesis testing (i.e., the way we do it with normally distributed data) must be re-evaluated when considering lognormal data.

\section{B-2.2 JUSTIfication fOR USING THE LOGNORMAL}

Empirically the lognormal distribution seems to fit radiological data from environmental settings. This may be justification in itself for its use. Also, an adaptation of the model described as the theory of breakage by Aitchison and Brown (1957) can be readily adapted to the diffusion of radionuclides or pollutants to provide a theoretical justification for the lognormal distribution. This is summarized by Blackwood (1990). 
Whatever theoretical basis exists for assuming a lognormal distribution for radionuclide or related data, pragmatic reasons for its use seem to dominate in the iiterature. The lognormal distribution has emerged as the most popular alternative to assuming normality most likely because it seems to fit most data sets adequately and because of the convenience it affords in data analysis. It is the next best thing to a normal distribution in the sense that, once the data is transformed, all the usual normal theory analysis methods apply. The following examples illustrate the pervasive empirical basis of the lognormal distribution for environmental data in general and radiological data in particular.

Eberhardt and Gilbert (1980), observing that frequency distributions of transuranic data are "ofton dramaticaliy skewed," recommend using "a logarithmic transformatiol. , the data before doing any statistical analysis involving significance tests based on the normal distribution." Georgopoulos and Seinfeld reported that "the two-parameter lognormal has been the most popular in representing air pollutant concentration data." Bernarie (1971) gives a justification for a lognormal distribution for ground level concentration of atmospheric dispersion of a single pollutant source in a single direction. He argues that all the generally accepted theoretical formulae for atmospheric dispersion indicate that ground level concentration is inversely proportional to the instantaneous wind speed. Furthermore, empirical observations have shown the distribution of wind speed to generally follow a lognormal distribution. So, since the inverse of a lognormal distribution is also lognormal, the ground level concentration should be lognormally distributed as well.

Perhaps the best description of the pragmatic use of the lognormal distribution comes from Dennis and Patil (1988) whose comments relative to general ecology studies also apply to radionuclides in the environment:

Possibly the main role of the lognormal in ecology is simply to serve as the handiest adjustable wrench in the toolbox of statistical distributions. Ecological abundance data are intrinsically positive, with a few enormously high data points 
typically arising in every study. The lognormal distribution is an ideal descriptor of such data, with a positive range, right skewness, heavy right tail, and easily computed parameter estimates.

If data are skewed and do not show a constant variance across groups with different mean values, often a logarithmic transformation is used simply to stabilize the variance and make the data symmetrical so that applying normal theory analysis methods seems more appropriate. The implicit assumption in this situation is that, since normal theory methods are being applied to the log transformed data, the original data must be approximately lognormal. However, as discussed earlier, a logarithmic transformation will only be successful in stabilizing the variance if the error term in the original underlying model is multiplicative. Eberhardt and Gilbert (1973) argue for a multiplicative error structure for environmental data by noting that, while means range over many orders of magnitude across different data sets, coefficients of variation stay relatively constant, making normally distributed additive errors unlikely.

\section{B-2.3 Properties of Lognormal Variables and Their IMPLICATIONS FOR STATISTICAL ANALYSIS}

\section{B-2.3.1 Mathematical Properties}

The lognormal distribution implies a multiplicative rather than an additive model for the data. This can be most easily seen by beginning with a measurement model which has additive, normally distributed, error or distributional terms. To legitimately use normal theory methods on data, a measurement model such as

$y_{i}=\mu+m_{i}$,

is assumed, where $y_{i}$ is the data value, $\mu$ is a mean value and $m_{i}$ is a random, normally distributed term with mean zero and variance $\sigma^{2}$ (denoted $N\left(0, \sigma^{2}\right)$ ). 
If such data are obtained through a logarithmic transformation of data, that is $y=\ln (x)$, then the original lognormal model must be of the form

$$
\begin{aligned}
x_{i} & =\exp \left(y_{i}\right) \\
& =\exp \left(\mu+m_{i}\right) \\
& =\exp (\mu) \exp \left(m_{i}\right)
\end{aligned}
$$

so the effects in the original lognormal model are multiplicative rather than additive. In other words, the variance of the $x_{i}$ is a function of $\mu$ in the lognormal model but not in the normal model.

In order to discuss the statistical properties of the lognormal distribution, some more rigorous mathematical notation is helpful. If $Y=$ $\ln (X)$ is normally distributed with mean $\mu$ and variance $\sigma^{2}$, then $X$ is said to be lognormally distributed with parameters $\mu$ and $\sigma^{2}$. The normal distribution of $Y$ is denoted $N\left(\mu, \sigma^{2}\right)$. Similarly $\operatorname{LN}\left(\mu, \sigma^{2}\right)$ denotes the Tognormal distribution of $X$. Some important properties of the lognormal distribution are

$$
\begin{aligned}
& \text { mean }=e^{\left(\mu+\frac{1}{2} \sigma^{2}\right)} \\
& \text { variance }=e^{2 \mu} e^{\sigma^{2}}\left(e^{\sigma^{2}}-1\right) \\
& \text { median }=e^{\mu} \\
& \text { skewness }=\left(e^{\sigma^{2}}+2\right)\left(e^{\sigma^{2}}-1\right)^{\frac{1}{2}} \\
& \text { kurtosis }=e^{4 \sigma^{2}}+2 e^{3 \sigma^{2}}+3 e^{2 \sigma^{2}}-3 \\
& \text { coefficient of variation }=\left(e^{\sigma^{2}}-1\right)^{\frac{1}{2}} .
\end{aligned}
$$


Parameters of a distribution can take many forms. Three basic types that relate to the various configurations the density function can obtain are location, scale, and shape parameters. In terms of a graph of the probability density function (e.g. Figures B-2.3.1 and B-2.3.2), a location parameter establishes the position of a distribution along the $x$ axis. Changing the location parameter is equivalent to adding a constant to every $x$ value, thus shifting a distribution left or right along the $x$ axis. Scale parameters are multiplicative and provide the measurement scale for the $x$ axis. Changing a scale parameter amounts to multiplying each $x$ value by a constant, shrinking or expanding the graph but not otherwise changing its shape. 'Shape parameters affect the general configuration of a distribution function in other ways than just changing the scale or location. Examples are parameters determining skewness and kurtosis.

For a normal distribution, $N\left(\mu, \sigma^{2}\right), \mu$ is the location parameter and $\sigma$ is the scale parameter. Normal distributions do not have shape parameters, thus the skewness and kurtosis are fixed for all normal distributions.

Unlike the normal distribution, the lognormal distribution has no location parameter but does have a shape parameter. It has no location parameter because it is always tied to zero, the minimum value. That it has a shape parameter means varying values of skewness and kurtosis can be obtained in lognormal distributions.

The scale parameter for a lognormal is $e^{\mu}$, the median of the distribution. Thus increasing $e^{\mu}$ in a lognormal distribution stretches the distribution in an analogous manner to increasing o in a normal distribution. This is illustrated in Figure B-2.3.1 which shows the probability density function for three lognormal distributions with varying $\mu$, but the same $\sigma^{2}$ value.

Increasing $\mu$ also increases the mean, mode, and median of the distribution, so is related to measures of central tendency. Skewness and kurtosis are constant for all three distributions in the figure, since they are functions of $\sigma^{2}$ alone. That is, any two lognormal distributions with the 


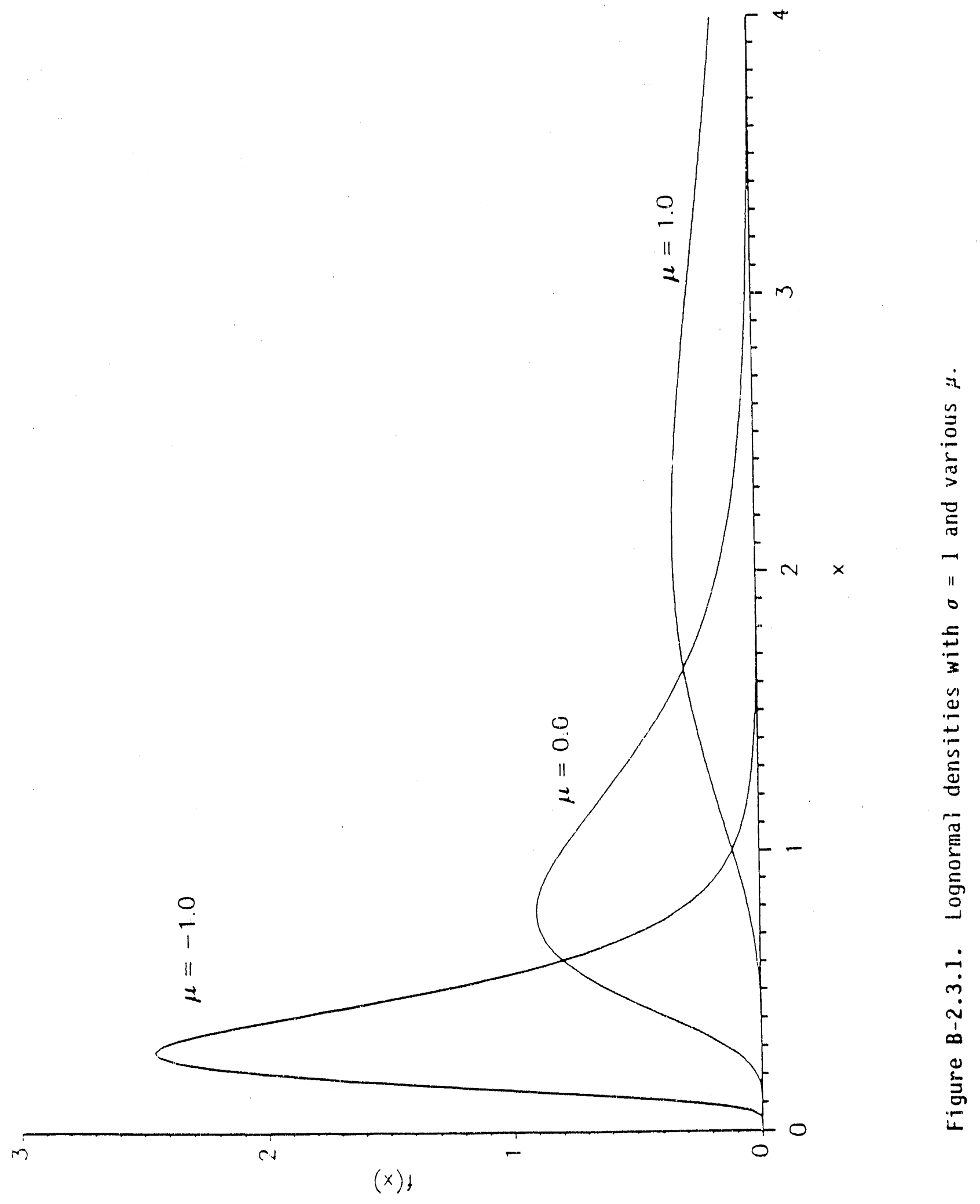




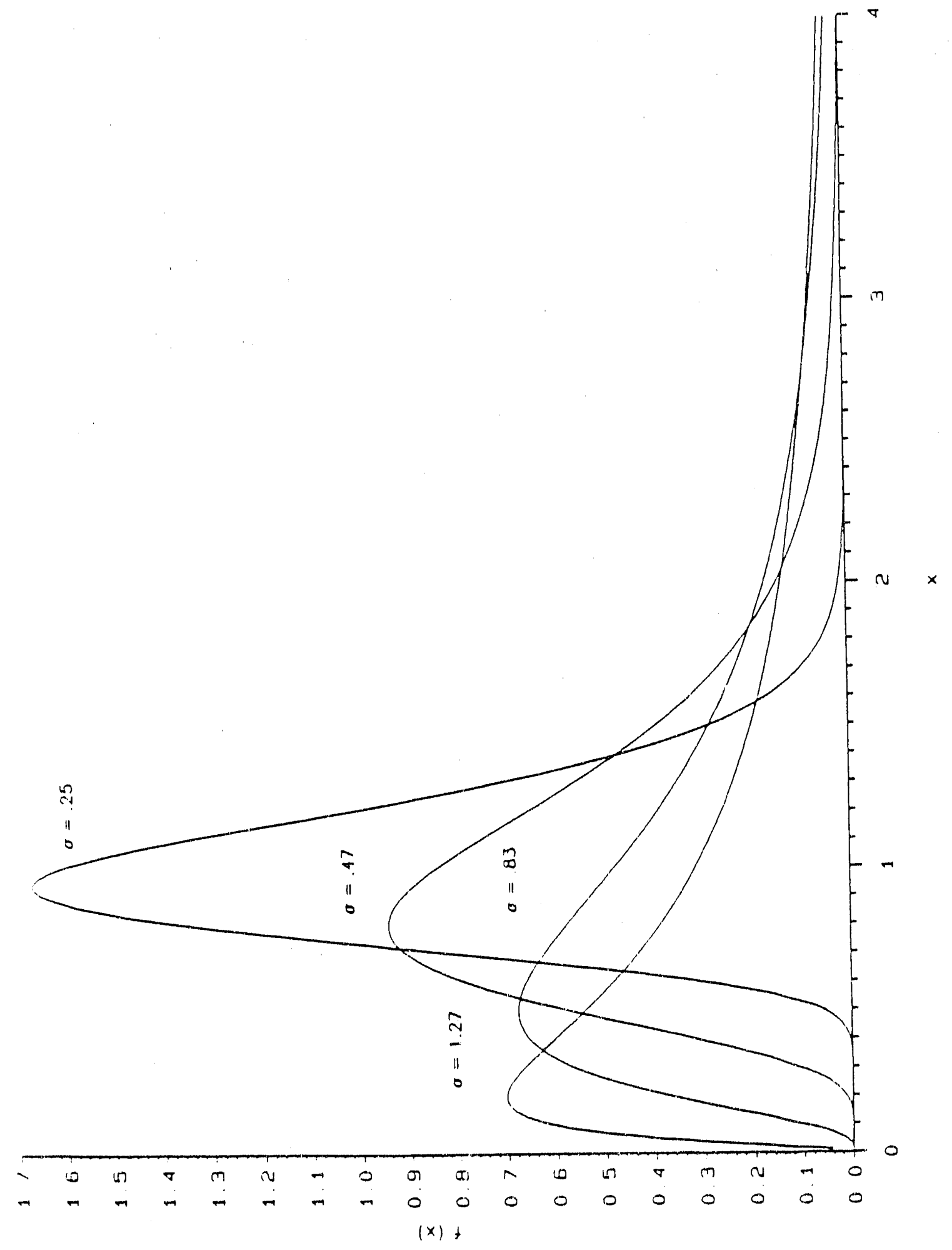

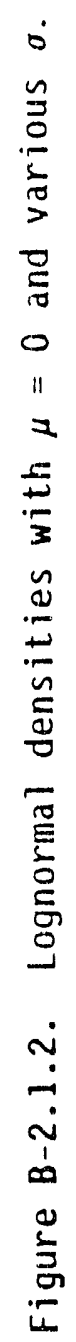


same $\sigma^{2}$ value but different $\mu$ values will still have the same shape (in the same sense that two normals with differing values of $\sigma^{2}$ have the same shape).

The shape parameter for the lognormal distribution is $\sigma$. The range of shapes obtainable with the lognormal distribution and the relationship of the parameters to the shape of the distribution can be observed in Figure B $\cdots 2.3 .2$, which show the graphs of several lognormal probability density functions for varying values of o but a constant $\mu$ value. The graphs illustrate that, the greater the value of $\sigma$, the more skewed the distribution and the heavier the tail (kurtosis). The coefficient of variation also increases with larger values of 0 .

Because the lognormal distribution is always positively skewed, the mode of a lognormal distribution is always less than the median which is in turn less than the mean. Note that for the smallest value of $\sigma^{2}$ in Figure B-2.3.2, the shape and symmetry of the lognormal might, at least at a casual glance, be mistaken for that of a normal distribution. The mode, median, and mean for this distribution are $.94,1.0$, and 1.03 respectively, indicating the near symmetry of the distribution. The skewness is .78 and kurtosis is 4.1 compared to the corresponding values of 0 and 3 for any normal distribution. For this distribution and a sample size of 25 , the probability of detecting a departure from normality is less than $20 \%$ (for a significance test with a $5 \%$ Type I error rate). This compares to a probability of essentially $100 \%$ when $\sigma^{2}=1.61$ (the most skewed distribution in the graph).

For any lognormal distribution the power for detecting departures from normality is a decreasing function of $\sigma^{2}$. This is true because, as $\sigma^{2}$ goes to zero, the standardized lognormal distribution tends to a standardized normal distribution (Shimizu and Crow, 1988). That the difference between a normal and lognormal distribution is hard to detect in small samples with small $\sigma^{2}$ values may explain why the normal distribution is sometimes found to provide an adequate fit to radiological data more typically expected to be lognormal. 


\section{B-2.3.2 Measures of Central Tendency}

Measures of central tendency are indicators of typical or average values used to summarize a distribution. The most common measures of central tendency are the (arithmetic) mean, median, and mode. For symmetric, unimodal distributions such as the normal, all three measures take on the same value, so there is no problem with choosing the most appropriate measure. For the lognormal distribution however, a decision must be made as to the best summary measure of central tendency. The choice will vary depending on what the purpose of measurement is.

The Mean. Summary statistics are often used to compute total dosage, lifetime exposure, the total amount of a constituent in a specific area, or other similar measurements. In this case an estimate of the mean of the untransformed data is the proper choice of summary value. Denote the population mean by $\alpha$. As noted above, $\alpha$ is a function of both $\mu$ and $\sigma^{2}$. Thus it is not purely a measure of central tendency in the same sense as the mean for a normal distribution is.

There are various ways of estimating $\alpha$, some better suited for particular purposes than others. Given the relationship of $\alpha$ to $\mu$ and $\sigma^{2}$, that is

$$
\alpha=e^{\left(\mu+\frac{1}{2} \sigma^{2}\right)},
$$

one method of estimation is to replace $\mu$ and $\sigma^{2}$ in this equation with the sample estimates from the transformed data,

$$
\hat{\mu}=\bar{y}=\frac{1}{n} \sum_{i=1}^{n} y_{i}
$$

and 


$$
\hat{\sigma}^{2}=\frac{1}{n} \sum_{i=1}^{n}(y-\bar{y})^{2} .
$$

This gives the maximum likelihood (ML) estimator for $\alpha$. (Note that $\hat{\sigma}^{2}$ is the ML estimator of $\sigma^{2}$ rather than the usual unbiased estimator which has $n-1$ rather than $n$ as the divisor.) This estimator has several desirable properties. Besides being easy to calculate, it approaches unbiasedness and minimum variance for large samples. However, for small samples, it can be extremely biased and can have infinite variance (Shimizu, 1988, p. 38). For further discussion, see Blackwood (1990).

The simplest reasonable estimator of $\alpha$ for the lognormal is the sample mean, the (arithmetic) mean of the untransformed sample data:

$$
\bar{x}=\frac{1}{n} \sum_{i=1}^{n} x_{i} .
$$

The sample mean is unbiased, but has a larger variance than the MLE. However, for smaller values of $\sigma^{2}$ and small sample sizes, the sample mean is nearly as efficient as the MLE. Koch and Link (1980) suggest using the sample mean for lognormal distributions whenever the coefficient of variation is estimated to be less than 1.2. For larger values of $\sigma^{2}$, the sample mean estimator still compares favorably when only the types of data. sets actually likely to be analyzed are considered (Blackwood, 1991).

The sample mean is unbiased regardless of the true underlying distribution of the data. Thus even if an error is made in specifying the distribution, or if the distribution is only approximately lognormal, the sample mean still retains its optimal property. This is not true of the other estimators. 
One caution in using the sample mean as an estimator of a needs to be mentioned. Even though the sample mean is unbiased for the lognormal distribution, it will underestimate a most of the time when small sample sizes are combined with large $a^{2}$ values. This result seems surprising at first, but it is an obvious result of the skewness of the distribution. For any distribution, the most likely value will be the mode, which will be considerably smaller than the mean in a highly skewed distribution. While an occasional large observation (much larger than the mode or the mean) will occur which brings the average mean calculation up sufficiently for the estimator to be unbiased, these large values will occur only rarely in small samples. So most of the time the sample mean will underestimate $\alpha$.

The Median. When evaluation of total exposure is not the purpose of calculating a summary measure, the median, $e^{\mu}$, may be a more reasonable measure of central tendency for the lognormal distribution. In addition to being a more "typical" value when the data are highly skewed, the median for the lognormal distribution is a function of $\omega$ alone so does not have the interpretation problem associated with $\alpha$. That is, the median can change without there being a corresponding change in the shape of the distribution (skewness, kurtosis, etc.).

The usual sample estimate of the median is the geometric mean,

$$
\bar{x}_{g}=e^{\bar{y}}=\left(\prod_{i=1}^{n} x_{i}\right)^{\frac{1}{n}} .
$$

This is the maximum likelihood estimator for the median and thus has desirable asymptotic (large sample) properties. However, the estimator is positively biased and for small samples could be misleading. (The estimate $\bar{y}$ is unibiased for $\mu$ but the exponential transformation, since it is not linear, produces a biased estimate of the median of the $x$ population.) 
The simplest estimator of the population median is just the samnle median of the $x^{\prime} s$. This nonparametric estimator will not be fully efficient for truly lognormal data. However, it is easy to calculate, considerably less sensitive to outiliers than the geometric mean, and it does not depend on the form of the population distribution.

\section{B-2.3.3 Measures of Dispersion}

It is tempting to relate the variance of the lognormal distribution, denoted here by $B^{2}$, to dispersion in the same manner as the variance of a normal distribution is used. However, as with the use of a described above, $B^{2}$ is related to both the scale and shape parameters of the lognormal so the analogy with the normal distribution is not strictly valid. Furthermore, the skewness of the distribution affects the interpretation. For a normal distribution, the variance relates directly to how far from the mean value an observation is likely to lie, in either direction. However in the lognormal case the probability of an observation occurring a fixed distance from the mean is different in one direction than it is in the other.

Because of the difficulties of interpretation, the value of $B^{2}$ as a measure of dispersion for a lognormal distribution is unclear (with the exception of lognormals that are close to being norma1). Alternative measures of dispersion include stating the value of $\sigma^{2}$, or calculating percentiles, interquartile ranges, etc. The geometric standard deviation, defined as $e^{\sigma}$, and the error factor (the ratio of the 95 th percentile to the 50th percentile) are used sometimes as well:

\section{B-2.3.4 Confidence Intervals and Hypothes is Tests}

Confidence intervals for functions of $\mu$ alone, such as the median $e^{\mu}$, can be easily calculated by making use of the normally distributed transformed data. If $(a, b)$ defines the usual normal theory confidence interval for $\mu$ based on the $\log$ transformed data (i.e. $\bar{y} \pm t s_{y} / n$, where $t$ is the appropriate Student's t value and $s_{y}$ is the standard deviation of the observed $y$ values), then an appropriate confidence interval for any monotone function $f(\mu)$ is just 
$(f(a), f(b))$. The interval for $f(\mu)$ will be optimal in the same sense that $(a, b)$ is optimal for $\mu$ (e.g. uniformly most accurate).

For the median the confidence interval is $\left(e^{a}, e^{b}\right)$. Note that the interval is not symmetric about the median estimate. That is

$$
e^{b}-e^{\bar{y}} \neq e^{\bar{y}}-e^{a}
$$

However, the ratios are equal,

$$
\frac{e^{b}}{e^{\bar{y}}}=\frac{e^{\bar{y}}}{e^{a}} .
$$

Functions of $\sigma^{2}$ alone are also calculated easily by referring to the chisquare distribution of $(n-1) s_{y}^{2} / \sigma^{2}$ and then transforming. Functions of $\sigma^{2}$ of interest might be the geometric standard deviation $\left(e^{\sigma}\right)$, the coefficient of skewness, the coefficient of kurtosis, a id the coefficient of variation.

Since the usual normal theory does not consider confidence intervals for joint functions of $\mu$ and $\sigma^{2}$, interval estimates for measures such as $\alpha$ and $\beta^{2}$, and for other functions of both $\mu$ and $\sigma^{2}$, are more difficult. Some methods are discussed by Blackwood (1990).

The usual advice for hypothesis tests involving lognormal variables is to transform the data and use standard normal theory methods. If there is no need to express results in the original scale, then no further changes in the standard methods are necessary. This means that a hypotheses that involves only $\mu$ or only o, such as a hypothesis about the median $e^{\mu}$, is most easily tested. For further discussion, see Blackwood (1990). 


\section{B-2.4 Discussion AND ReCOMMENDATIONS}

\section{B-2.4.1 Use of the Logarithmic Transformation}

In terms of making the most use of standard normal theory methods, the most straightforward method of dealing with lognormal data is to do a logarithmic transformation of the data and state all estimates, hypotheses, and test results in terms of the transformed data. Then everything proceeds as in the usual normal case. Eberhardt and Gilbert (1980) recommend that, if the results of statistical tests are of primary interest, then they should be given in terms of the transformed data.

If it is not practical to discuss everything in terms of the transformed data, in particular if at least some statement of results in the original scale are required, then stating required estimates and tests in terms of the median of the discribution affords the most direct analysis. Normal theory methods can be used for estimates and tests of the median with only minor modification (e.g. stating hypotheses in terms of ratios, and getting confidence intervals not symmetric about the estimate).

If the mean on the lognormal scale (or other compound functions of $\mu$ and $\sigma^{2}$ ) is of interest, methods other than those based on normal theory are required. Two possibilities are maximum likelihood estimates (and likelihood ratio tests), or nonparametric estimators such as the sample mean.

\section{B-2.4.2 Alternatives to the Lognormal Distribution}

Application of the lognormal distribution to certain classes of data have been so common that researchers sometimes fail to consider other distributions that might work better. The logrormal assumption is often just one of convenience for positive highly skewed data. It allows some justification for using normal theory methods but the required assumptions of multiplicative errors etc., may not be consistent with the true underlying distribution. Even if multiplicative errors apply, the transformed data may still be far 
from normal so that even appealing to the robustness of certain normal theory methods may not make sense. In that case, other distributions or methods may provide a more reasonable alternative.

Variations of the two-parameter lognormal are sometimes appropriate. The delta lognormal, which allows for zero values, is useful in certain applications, such as when there is no naturally occurring radiation so there can be true zero values in samples. The three parameter lognormal applies in cases where there is an unknown minimum value other than zero. While betweensample variability in radiological measurements may be lognormally distributed, individual radiological measurements are basically count data usually thought to follow a Poisson distribution. So a Poisson-lognormal mixture distribution may be useful in some applications as well.

Other parametric distributions can also be used successfully with positive, skewed data. Eberhardt and Gilbert (1973) studied the gamma distribution in relation to the distribution of radionuclide concentrations in biota and substrates. They reported some empirical evidence that the gamma distribution provides a better fit than the lognormal. Speer and Waite (1975) fit Weibull distributions to radiological environmental surveillance with some success. Johnson and Symons (1980) reported a better fit of the Weibull compared to the lognormal when applied to a set of ambient air quality data.

Nonparametric methods should also be considered as an alternative to assuming lognormality. In the analysis of weather modification data, one area where the lognormal distribution has been traditionally assumed, nonparametric methods are now preferred due to their wider range of validity (Crow, 1988).

\section{B-2.4.3 Robustness of Lognormal Analysis Methods}

Given that lognormal methods are often used on pragmatic grounds, it is of interest to consider what happens when they are used on data that are in fact from other distributions. In a study of censored data analys is methods, Gilliom and Helsel (1986) found that estimation methods based on assuming lognormality worked well for data from gamma Jistributions, delta lognormal 
distributions, and mixtures of two lognormal distributions. Blackwocd (1991) found that lognormal methods produced acceptable results when used is normally distributed data. Eberhardt and Gilbert (1973), using simulations to compare the performance of F-tests, concluded that assuming either the gamma or lognormal distribution provide about the same levels of power and robustness regardless of which of the two distributions the data actually originated from. They concluded that assuming lognormality for convenience has little penalty in terms of accuracy of results. 


\section{B-3 ONE SAMPLE}

\section{B-3.1 Point Estimates and Confidence Intervals}

Two estimation methods are presented in the subsections below, maximum likelihood (ML) estimation and the LR method. These two methods give the best estimators, according to the extensive simulations of Gilliom and Helsel (1986). Two methods not considered in those simulations are the bias-adjusted ILE with censored data (Cohn, 1988), and linear combinations of order statistics (Gupta, 1952, and Sarhan and Greenberg, 1962). The bias adjusted MLE is mentioned below in the discussion of the MLE. Linear combinations of order statistics are discussed here:

Linear combinations of order statistics are estimators of the form $\mu^{*}=\sum \beta_{i} X_{(i)}$ $\sigma^{*}=\sum \gamma_{i} X_{(i)} \quad$.

Values of the coefficients $\beta_{i}$ and $\gamma_{i}$ are tabulated by Gupta (1952) and Sarhan and Greenberg (1962), for various levels of censoring. The coefficients are optimal in the following sense: among all estimators that are linear combinations of order statistics, the coefficients presented give the minimumvariance unbiased estimates, under Type II censoring. Although the estimators can be used with Type I censoring, they are biased estimators in this case, and have larger MSEs than the MLEs have (Blackwood, 1989). Therefore they are not recommended for use with Type I censored data, and are not considered further here.

\section{B-3.1.1 Maximum Likelihood Methods}

Maximum Likelihood Estimation (MLE) methods work reasonably well for larger sample sizes and smaller variances. They work badly for small sample sizes and large variances. It is likely that in this case there will sometimes be estimators of $\sigma^{2}$ that have infinite variance (this is known to be true in the case of zero censoring). Maximum likelihood estimation for censored data is easily implemented with existing survival analysis programs. 
These programs generally allow a number of distributional assumptions. For example, the LIFEREG procedure in SAS allows the Weibul1, exponential, gamma, normal, lognormal, logistic, and loglogistic distributions.

For lognormal data, adjustments are available for MLE estimates for censored data that make them nearly unbiased minimum variance estimates. (Cohn, 1988) They are relatively difficult to implement, although FORTRAN programs for their calculation are available. Moreover, the bias of the MLE is only substantial when estimating moments of a strongly skewed distribution, in which case we do not recommend estimating moments a 11. Therefore the biasadjusted MLE is not considered further here.

\section{B-3.1.2 The LR Method}

The LR method is recommended by Gilliom and Helsel (1986). Although the method can be thought of informally as a graphical method, a more precise calculational procedure is needed for computer simulations. This more precise method is given here.

Denote the observations by $x_{1}, \ldots, x_{n}$, of which $c$ are censored, that is, $c$ are below the detection limit. Order the observations as $x_{(1)}, \ldots, x_{(n)}$, with the uncensored observations being $x_{(c+1)} \leq \ldots s x_{(n)}$. Let $\Phi$ denote the standard normal cumulative distribution function, tabulated in any statistics book. $\Phi(x)$ takes values from 0 to 1 as $x$ goes from $-\infty$ to $\infty$. Let $\Phi^{-1}$ be the inverse function; for example $\Phi^{-1}(0.95)=1.645$, and $\Phi^{-1}(0.025)=-1.96$. For each uncensored observation $x_{(i)}$, compute the normal score $z_{i}=\Phi^{-1}[i /(n+1)]$. We assume that the observations come from a lognormal population, that is, that the values of $\ln (x)$ come from a normal population. For the uncensored observations, perform a least squares fit of $\ln \left(x_{(i)}\right)$ on $z_{i}$; this can be done with a commercial statistics package as a simple linear regression of $\ln (x)$ on $z$. Graphically, the values of $z$ are thought of as plotted on the horizontal axis and the values of $\ln (x)$ on the vertical axis. The fitted line passes approximately through the points. Now use the fitted line to extrapolate back and find the values of $x$ that correspond to $z_{1}$ through $z_{c}$. The actual values are unknown, but less than the detection limit; therefore 
use these calculated values, based on extrapolating the fitted line, instead. Take the entire set of $x$ values, $c$ calculated and the rest observed, and calculate the sample mean and the sample variance. These are the LR estimates of the population mean and variance. An example using SAS is presented in Appendix A. 


\section{B-4 TWO SAMPLES}

Four methods were evaluated for their ability to test for equality of means (medians for nonparametric tests) in the presence of censored data. Three of the techniques, the detection limit test. (DL test), the Wilcoxon rank sum test (also known as the Mann. Whitney, Wilcoxon test, abbreviated MWW), and the Prentice test, are nonparametric techniques. The use of the latter two tests in the presence of censored data is described in Millard and Deveral (1988). The fourth technique, Iterative Least Squares, uses an iterative algorithm based on least squares regression techniques. It is most fully explained in Schmee and Hahn (1979).

The MWW test, Prentice test, and ILS test all perform well and have approximately the sant power to detect differences. These tests are also capable of handling multiple detection limits and comparisons of more than two groups (we only.consider two groups here because it is our experituce that it is the most common situation in environmental practice). However, of the three test.s, the ILSS test is likely to be the most difficult to implement in a statistical computing package. The MWW test is widely available in most statistical packages and is easily modified to accuunt for censoring when there is a single detection. It requires a little more work when there are multiple detection limits, but this should still be possible in most statistical packages. Hence, for its simplicity, availability, and performance, the MWW test may be recommended for general use.

\section{B-4.1 TwO-SAMPle TeSting: NonPARAMETriC MetiodS}

Simulations were run on three different nonparametric 2-sample comparison tests. The three tests are 1) the Wilcoxon test, 2) the Prentice test, and 3) the DL test. A fourth test, the Runs test, was discontinued due to a poor showing in the early rounds (and to make more efficient use of my computer's time).

The Wilcoxon test (also called the Mann-Whitney rank sum test) ranks all the data alld the:l sums the ranks within each sample. The greater the difference between the sums of the ranks, the more likely the two samples have different means or medians. This test will be denoted the MWV test for the remainder of this report. Details of the test can be found in Hollander and Wolfe (1973). As applied to censored data, the test is discussed in Millard and Deveral (1988). For this simulation, a normal approximation was used to compute probabilities (see Steel and Torrie, 1980).

The Prentice test is based on computation of a linear combination of the rantis of the data. The choice of coefficients used in the linear equation is selected based on the distribution of the datin in 
urder to maximize the power of the test. In this sense, the technique is not truly nonparametric. The test is related to the Wilcoxon test. Details can be found in Millard and Deveral (19sis). For (his, simulation, normal scores (see Table 5 of Millard and Deveral, 1988), and the permutation variance. (see equation 10 in . Millard and Deveral) were used, as ivas a normal approximation 10 nompute probabilities.

The DL test (detection limit test) is essentially the same as the median test, using the detection limit as the break point rather than the median. For more detail on the median test, see Steel and Torrie (1980). For this simulation, the exact hypergeometric probability was computed.

\section{B-4.1.1 Simulation Methods}

A sample of size 20 was selected from a pseudo-standard normal distribution (with mean 0 and variance 1). The first ten pseudo-random numbers were assigned to the first group. The latter ten had their values shifted by addition of the mean, which took values $0,0.5,1.0$, and 1.5 , and were assigned to the second group. 500 samples were so constructed.

Each sample was censored at the 20,40,60, and $80 \%$ point of the joint density function of the two groups. For each censoring level, a single censoring point was calculated. Any observation falling below that point was censored. If all the observations fell below the censoring level, then that sample was not analyzed. This occurred about $1 \%$ of the time for the $80 \%$ censoring level, and occurred once at the $60 \%$ censoring level.

At each censoring level, the three test statistics and probabilities of a larger value were calculated. The null hypothesis was of equal medians, the alternative hypothesis was that the second group median was larger than the first. Hence, all tests were against a one-sided alternative. This onesided alternative was used to mimic the common practice in environmental monitoring of comparing contaminated areas to background. In these circumstances, it is generally not of interest to test if the concentration in contaminated areas is less than background.

All sample generation and calculations were performed using S/PC (Technology Partners, Inc., Orem, Utah). The computing was done on an IBM PS/2 Model 70.

\section{B-4.1.2 Discussion}

Simulation results are shown in Table B-4.1.1. As can be seen, the DL lest performed poorl! compared to the other two tests in terms of both power and maintained $\alpha$-level (i.e. $5 \%$ rejected at $\mu$. $=1)$. The Prentice test performed slightly better than the MWW test in terms of power, but not quite as well as the MWW test in terms of maintained $\alpha$-level. Generally, for the MWW test and the 
Table B-4.1.1. Nonparametric 2-Sample Test Simulation Results

\begin{tabular}{|c|c|c|c|c|c|}
\hline \multirow[b]{2}{*}{$\mathrm{n}$} & \multirow[b]{2}{*}{$\mu_{2}$} & \multirow{2}{*}{$\begin{array}{c}\% \\
\text { Censored }\end{array}$} & \multicolumn{3}{|c|}{$\%$ Rejected $^{a}$} \\
\hline & & & Wilcoxon & Prentice & DL test \\
\hline \multirow[t]{4}{*}{10} & 0 & 20 & 4.6 & 5.4 & 2.0 \\
\hline & & 40 & 4.6 & 6.4 & 1.6 \\
\hline & & 60 & 6.4 & 7.2 & 2.6 \\
\hline & & 80 & 5.9 & 6.5 & 1.2 \\
\hline \multirow[t]{4}{*}{10} & 0.5 & 20 & 30.4 & 33.2 & 6.8 \\
\hline & & 40 & 28.4 & 33.8 & 12.8 \\
\hline & & 60 & 26.1 & 30.0 & 13.6 \\
\hline & & 80 & 17.2 & 21.2 & 8.5 \\
\hline \multirow[t]{4}{*}{10} & 1.0 & 20 & 65.6 & 69.2 & 18.2 \\
\hline & & 40 & 62.8 & 67.2 & 35.2 \\
\hline & & 60 & 60.6 & 65.2 & 37.4 \\
\hline & & 80 & 46.7 & 51.1 & 24.0 \\
\hline \multirow[t]{4}{*}{10} & 1.5 & 20 & 93.8 & 94.7 & 40.0 \\
\hline & & 40 & 92.7 & 93.8 & 69.1 \\
\hline & & 60 & 86.5 & 90.4 & 70.2 \\
\hline & & 80 & 64.1 & 66.9 & 42.7 \\
\hline
\end{tabular}


Prentice test, as the censoring level increased, the power decreased. For the DL test, power wis consistently highest at the $60 \%$ censoring level. As would be expected, the power of the tests increased as the difference between the means of the two groups increased.

Simulation results are only reported for samples of size 10 for each group. Some simulation was done with samples of size 20. The results from these latter simulations showed a signiticant increase in the power of the test,s, particularly the DL test and for all 3 tests when the differences between means was small $(0.5)$.

The poor performance of the DL test with respect to the other two tests is not surprising since it uses less information contained in the sample. However, it was hoped that at high censoring levels, the DL test would compare favorably to the other two tests. This was not the case. Specifically, when the mean of the second group was 0.5 or 1.0 , the DL test was approximately half as poiverful as the other two tests at the $80 \%$ censoring level.

Because of symmetry in the hypergeometric statistic on which the DL test is based, ic was expected that the power of the DL test would be symmetric about $50 \%$ censoring. However, the results show that the power is slightly higher for censoring levels above $50 \%$ than for the corresponding censoring level below $50 \%$. The DL test was also poor at maintaining the significance level, as can be seen from the results when the two group means are equal. This makes this test a conservative test. Based on these results, there is no reason to recommend the DL test over the remaining two tests.

The Prentice test was consistently more powerful than the MWW test. However, since the samples were from normal distributions, the simulation was slanted in favor of the Prentice test based on normal scores. From this simulation, it is unclear whether the Prentice test would have still been more powerful than the MWW test if the data had come from a non-normal distribution. Further, the Prentice test also did not maintain the $5 \%$ significance level when the null hypothesis was true. Rather it maintained approximately a $6 \%$ significance level. On the other hand the $\mathrm{MWW}$ test was closer to maintaining the $5 \%$ level.

Based on these simulations, use of either the MWW or Prentice test could be recommended, particularly if the data is normally distributed. On the other hand, if the data is known to be nonnormal, one should probably use the MWW statistic.

Another apparent reason to recommend either the $\mathrm{MWW}$ or Prentice test is that they have been modified to allow for multiple detection limits, as discussed in Millard and Deveral (1988). It is common in chemical analytical data to have multiple detection limits due, for example, to dilution of the original sample. However, few statistical techniques allow for multiple detection limits. Millard and Deveral (1988) give some simulation results of these methods in the presence of multiple detection 
limits and get mixed results, dependent on the parent distribution.

\section{B-4.2 TwO-SAMple TESTING: I'TERATIVE LEAST SQUARES}

It frequently occurs in environmental sampling that two samples are independently collected and compared to see if both samples are from the same population. The most common example is comparison to background. There are a number of ways of making the comparison in the case when data are uncensored. The most commonly used methods involve comparing a measure of location from each sample. In the parametric case, the location measure is typically the mean. In the nonparametric case, it is the median. In this section, a method is given for comparing two samples by using a linear model and least squares analysis in the presence of censored data. The method involves iterations and has been called iterative least squares (ILS) by Schmee and Hahn (1979), but is based upon the widely used EM algorithm (Dempster, Laird, and Rubin, 1979). We will use he term ILS as it is more specifically descriptive of the technique.

The model that is used to describe the data is

$y_{i j}=\mu+\beta_{i}+\varepsilon_{i j}$

where $i=1,2$ is a group indicator, $j=1(1) n_{i}$, and $n_{i}$ is the number of observations in the $i$ 'th group. In this parameterization, the $\beta_{i}$ is the parameter of most interest as it represents the group effect (often called the treatment effect in the standard terminology of experimental design). If there is a significant difference between the $\beta_{i}$, then there is evidence that the two samples are not from the same population.

\section{B-4.2.1 Specification Of The Method}

Consider the model

$y=\mu+\beta_{1} x_{1}+\epsilon$

where $y$ is the response variable, $x_{1}$ is the grouping variable, $\epsilon$ is the random error term (residual), and $\mu$ and $\beta_{1}$ are the parameters to be estimated. It is assumed that the expected value of $c$ is 0 and the variance is $\sigma^{2}$. We first review the regression calculations for the uncensored case.

If $\mathrm{n}=\mathrm{n}_{1}+\mathrm{n}_{2}$ independent observations of the response are taken for $\mathrm{n}$ realizations of the set of $x$ 's, then for each observation the model is 
$y_{i}=\mu+\beta_{1} x_{1 i}+\epsilon_{i}, \quad \mathrm{i}=1(1) \mathrm{n}$.

In this model, the $y_{i}$ and $x_{1}$ are known, while $\beta_{1}$ and the $\epsilon_{i}$ 's are unknown and must be estimated. The model (B4.2-3) with $n$ observations can be rewritten in matrix form as

$y=X \beta+t$

where $y$, the vector of response values, and $t$, the vector of residuals, are $n \times 1$ vectors, $X$ is an $n \times 2$ matrix with the first column being a column of l's and the second column being an indicator (grouping) vector of 0 s and 1 depending on whether the corresponding observation is taken from group 1 or 2, respectively. $\beta=\left(\mu, \beta_{1}\right)^{\mathrm{T}}$ is the vector of coefficients. The expected value of $\epsilon$ is 0 with dispersion matrix $\sigma^{2} \mathrm{I}$

The vector $\mathrm{y}$ and matrix $\mathrm{X}$ in Eqn. (B4.2-4) are known from the $\mathrm{n}$ observations. The estimate of $\beta$ is found as

$\widehat{\beta}=\left(X^{T} X\right)^{-1} X^{T} y$.

And the residuals are given by

$\widehat{\epsilon}=y-X \widehat{\beta}$.

The variance of the residuals, $\sigma^{2}$, is estimated as

$\widehat{\sigma}^{2}=\frac{\widehat{\epsilon}^{\mathrm{T}} \widehat{\epsilon}}{\hat{N}-2}$

Finally, an important property of the estimate $\widehat{\beta}$ is the variation in the estimation. This is contained in the estimated dispersion matrix, $\operatorname{va} r(\widehat{\beta})$, which is calculated as

$\operatorname{vâ} r(\widehat{\beta})=\widehat{\sigma}^{2}\left(X^{\mathrm{T}} \mathrm{X}\right)^{-1}$

The structure of $\operatorname{var}(\widehat{\beta})$ is 
$\operatorname{varr}(\hat{\beta})=\left[\begin{array}{cc}\operatorname{vâr}(\hat{\mu}) & \operatorname{covv}\left(\hat{\mu}, \hat{\beta_{1}}\right) \\ \operatorname{cov}\left(\hat{\mu}, \hat{\beta_{1}}\right) & \operatorname{vâr}\left(\hat{\beta_{1}}\right)\end{array}\right]$.

A simple way of assessing whether there is a difference between groups is to test whether $3_{1}$ is zero. This can be done with a t-test by dividing the estimate of $\beta_{1}$ by its associated standard error. That is,

$i=\frac{\hat{\beta_{1}}}{\sqrt{\operatorname{varr}\left(\hat{\beta_{1}}\right)}}$.

where $\hat{\beta_{1}}$ and $\operatorname{var}\left(\hat{\beta_{1}}\right)$ are calculated as shown in Eqns. (B4.2-j) and (B4.2-8), respectively. The t-value has degrees of freedom $n-p-1=n-2$, since $p$ is the number of independent (grouping) variables. This calculated $t$-value is compared to the tabled t-value at the appropriate significance level.

Iterative Least Squares. The ILS algorithm essentially requires that one put in replacement values for the censored data points and compute $\widehat{\beta}$ and $\widehat{\sigma}^{2}$. With these estimates, one then linds new replacement values for the censored data. These new estimates of the censored points are then used to recompute $\widehat{\beta}$ and $\widehat{\sigma}^{2}$. The iterations proceed to convergence.

The initial replacement values for the censored data are typically taken as the censoring points (detection limits), $\mathrm{DL}_{\mathrm{i}}, \quad \widehat{\beta}$ is then computed by Equation B4.2-5. The residual variance is then computed using a method analogous to Equation B4.2-7, but that accounts for the censoring. IVe identified three different estimates for the residual variance in the presence of censoring in the literature. The first, due to Schmee and Hahn (1979) is to use Equation B4.2-T with the censored observations represented by their replacement value. The.second method, identified by Aitien (1981). is to calculate the residual variance as

$$
\hat{\sigma}^{2}=\frac{\sum_{i \in u}\left(y_{i}-\widehat{\mu}_{i}\right)^{2}}{\left(n-c-\sum_{i \in c} \widehat{z}_{i} S\left(\widehat{z}_{i}\right)\right)}
$$

where

$$
\begin{aligned}
& i \in u=\text { all uncensored observations, } \\
& i \in c=\text { all censored observations, } \\
& n-c=\text { the number of uncensored observations, } \\
& \widehat{\mu}_{i}=\widehat{\mu}+\widehat{\beta}_{1} x_{11}, \\
& \widehat{z}_{i}=\left(\widehat{y}_{i}-\widehat{\mu}_{i}\right) / \widehat{\sigma},
\end{aligned}
$$




$$
\begin{aligned}
& \hat{y}_{1}=\widehat{\mu}_{1}-\widehat{\sigma} S\left(\left(y_{i}-\widehat{\mu}_{i}\right) / \widehat{\sigma}\right), \\
& S(v)=\phi(v) /[1-\Phi(v)] .
\end{aligned}
$$

Here $\Phi$ is the standard normal cumulative distribution function and $\phi$ is the corresponding density. The third method for estimating the residual variance is due to Wolynetz (1979b). In this case,

$$
\widehat{\sigma}^{2}=\frac{\sum_{i=1}^{n}\left(\widehat{y}_{i}-\widehat{\mu}_{i}\right)^{2}}{n-c+\sum_{i \in c} T\left(-\hat{H}_{i}\right)}
$$

where

$$
\begin{array}{ll}
\hat{y}_{i}= \begin{cases}y_{i} & \text { if } i \in u, \\
\hat{\mu}_{i}-\widehat{\sigma} S\left(-\widehat{H}_{i}\right) & \text { if } i \in c,\end{cases} \\
\widehat{H}_{i}=\left(D L_{i}-\widehat{\mu}_{i}\right) / \widehat{\sigma}, \\
T(v)=S(v)\{S(v)-v\},
\end{array}
$$

and all other symbols are as above. We also considered the denominator in Equation B4.2-11 with all adjustrnent for the number of parameters in the nodel. Note that the definition of $\hat{y}_{i}$ in Equation B4.2-11 differs from that defined in Equation B4.2-10. The form of the calculation of the replacement values given in B4.2-11 was used regardless of the method for estimating $\widehat{\sigma}^{2}$, since it was not clear in Aitkin's paper what the value of $y_{i}$ for censored data is in the equation for $\hat{y}_{i}$.

Finally, two different estimates of $\operatorname{Var}(\widehat{\beta})$ were used. The first estimate is given in Equation B4.2-8, with $\widehat{\sigma}^{2}$ calculated using one of the techniques given above. The degrees of freedom associated with the t-test given in Equation B4.2-9 was considered to be the denominator of the estimate of $\widehat{\sigma}^{2}$ rounded to the nearest integer.

The second method uses the asymptotic esti ate of $\operatorname{Var}(\widehat{\beta})$, due to Wolynetz (19T9b). The asymptotic information matrix, $G(\beta)$, is first estimated. Then $\operatorname{Var}(\widehat{\beta})$ is estimated as the inverse of the estimated information matrix. The elements of the information matrix, $\mathrm{g}_{j k}$, are given by

$g_{j k}=\hat{\sigma}^{-2}\left\{\sum_{i \in u} x_{i j} x_{i k}+\sum_{i \in c} x_{i j} x_{i k} T\left(-\hat{H}_{i}\right)\right\}$,

where $x_{1}$ represents the element in the matrix $X$ corresponding to the ${ }^{\circ}$ th row and $j$ th column. When using the asymptotic estimate of $\operatorname{Var}(\hat{\beta})$ for testing $\beta_{1}=0$, the degrees of freedom were assumed :0 be $\infty$, and hence the standard normal distribution was used rather than the t-distribution. 


\section{B-4.2.2 Simulations and Computing Methods}

Because of concerns expressed in Schmee and Hahn (1979) and Aithin (1981) with respect to bias in the estimate of the residual variance, simulations wero implemented to see if it was possible to come up with an unbiased estimate of the variance. There was also a need to look at Wolynetizs (1979a, 1979b) estimators for the variance, as these had not been considered in previous simulations evaluating performance.

The simulations were done in three parts. The first part was used to determine if any methods could be "thrown out" immediately as they did not perform well versus the other methods. The methods considered involved different estimates of the variance and were due to Aitkin (1981), Schmee and Hahn (1979), and Wolynetz (1979b). Under the latter estimates wo adjusted the degrees of freedom for the model parameters being estimated. The first set of simulations involved up to 500 runs.

The second set of simulations looked only at Wolynetz's (1979b) method for estimating the variance with a variety of degrees of freedom adjustments. The general form of the variance is $\hat{\sigma}^{2}=\frac{\sum_{i=1}^{n}\left(\hat{y}_{i}-\widehat{\mu}_{i}\right)^{2}}{n-c+\sum_{i \in c} T\left(-\hat{H}_{i}\right)-k}$,

where $k=0(1) 3$ is the adjustment factor studied. Equation B4.2-13 is identical to Equation B4.2-11 when $k=0$. The hypothesis test of $\mathrm{H}_{0}: \beta_{1}=0$ was tested using the non-asymptotic t-test shown in Equation B4.2-9. This set of simulations used 1,000 runs.

The third set of simulations extended the second set and included two testing methods: the non-asymptotic t-test of the hypothesis $\beta_{1}=0$ and the asymptotic version of che same test using the standard error of $\beta_{1}$ as given by Wolynetz (1979b). The variance shown in Equation B4.2-13 was used with two different values of $k, 0$ and 2. Thus, the third set of simulations actually encompassed fonr methods: non-asymptotic test with the variance estimator's denominator adjusted by $k=0$, nonasymptotic test with $k=2$, asymptotic test with $k=0$, and asymptotic test with $k=2$. The exact form of the non-asymptotic test is given in Equation B4.2-9, where the resulting t-value is compared to a t-distribution with degrees of freedom corresponding the nearest integer value of the denominator of the residual variance. The exact form of the asymptotic test is

$z=\frac{\hat{\beta}_{1}}{\sqrt{G(\hat{\beta})_{22}^{-1}}}$,

where $G(\hat{\beta})_{22}^{-1}$ is the second diagonal element of the inverse of the information matrix, (ii $\left.\bar{\beta}\right)$. 
Calculation of the elements of $\mathrm{G}(\hat{\beta})$ is shown in Equation B4.2-12. The third set of simulations used 5.000 runs.

The first set of simulations will not be discussed in detail here. They were run using an $S / P("$ program, which proved to be very time consuming. It immediately picked up problems with Aitkin's method, as the denominator of the variance estimate frequently went below zero for 60 and particularly. 80 percent censoring. Schmee and Hahn's method showed significant bias when estimating the residual standard deviation.

Simulation Settings. Sampling was from two normal populations, corresponding to two groups. The first population had mean 0 and variance 1 . The serond population had its mean varied ac 0, 0.5. and 1.0 and its variance constant at 1. The censoring was done at 20,40,60, and 80 percent levels of the joint population density function. That is. the censor points (i.e. detection limits in environmental data) were determined such that when considering both populations as one 20,40,60, and 80 percent of the data would be censored. Thus oniy one detection limit was assumed to be in effect. This may not be realistic as most environmental data sets have multiple censoring levels (note, though, that these techniques can all handle multiple censoring levels). Also two sample sizes were considered. In the first, 10 observations were taken from each group. 20 observations were taken from each group in another set of simulations. A simulation was dropped when there were less than 2 uncensored observations.

Computing Methods. Apart from the first set of simulations, which used S/PC, the simulations were run using a Pascal program compiled in TurboPascal. The regression algorithm is due to Givens (Kennedy and Gentle, 1980, p. 273). The random number generator has two parts. The first part used a linear cungruential generator (LCG) that produced uniform random numbers between zero and one. The LCG coefficients had previously been tested and found to be adequate. The unitorm pseudorandom numbers were then transformed to normal random variates using the Box-Mluller method.

For each setting of $\beta_{1}$ (i.e. 0, 0.5, and 1.0), up to 5000 data sets were produced. Each data set was then censored at the 20,40,60, and 80 percent levels. Deperding on the set of simulations, there were up to 5 estimates of the residual variance used on each data set and censoring level. In the final set of simulations. there were also two tests of $3_{1}=0$.

There were two convergence criteria. both of which had to be met. First, the square of the difference between two successive estimates of the residual variance had to be less than 0.00001 . Second the sum of the square of the difference between two successive estimates of $\mu$ and the square of the difference Detween two surcessive estimates of $3_{1}$ had to be less than 0.00001 . If the program did B- 34 
not converge in 100 iterations, the iterations stopped and the most current estimates were used. This was only a problem at the $80 \%$ censoring level. Since a large fraction of the $80 \%$ results are based on simulations that were not allowed to converge, they may be suspect. However, it is also typically true that by the end of 100 iterations the estimates are stable (i.e. there are no gross differences between the estimates from one iteration to the next).

\section{B-4.2.3 Results}

The various ILS methods were evaluated through a number of criteria. With respect to testing for a difference between two groups, the methods were judged based on their ability to maintain the $\alpha$ level when $\beta_{1}=0$ and their power when $\beta_{1}>0$. The $\alpha$-level was set at $5 \%$. It controls the Type I error rate; i.e. it establishes the allowable frequency of rejecting the null hypothesis that $\beta_{1}=0$, when in fact the null hypothesis is true. When the null hypothesis is false $\left(\beta_{1}>0\right)$, the Type II error rate measures the frequency of inrorrectly accepting the null hypothesis. The bias, standard deviation, and square root of the mean square error were considered when comparing the methods' ability to estimate $\mu, \beta_{1}$, and $\sigma^{2}$.

The first set of simulations (which used up to 500 runs) involved comparing the performance of ILS when using Schmee and Hahn's variance estimate, Aitkin's variance estimate, and Wolynetz's estimate. These simulations showed Wolynetz's method to be the most stable, that is, the estimator had the smallest variance. Aitkin's estimate produced negative variances at high censoring levels. Schmee and Hahn's method was not stable at high censoring levels, but performed well at low censoring levels. The Wolynetz estimate was found consistently underestimate the residual variance. Since Wolynetz makes no adjustment for the number of parameters estimated, it was decided to look at adjusting the denominator of the residual variance in the Wolynetz estimate. It was hoped that this might remove some of the bias in the estimate.

In the second set of simulations (which used 1,000 simulations) Wolynetz's residual variance estimator was adjusted by subtracting $0,1,2$, and 3 from the denominator. The results of the simulations in terms of \% Rejected are shown in Table B-4.2.1. Yone of the adjustments were able to maintain a $5 \%$ a!pha level at the 60 and $80 \%$ censoring levels. However, when the cisnominator was adjusted by 3 , the Type 1 error rate remained below 8\%; when adjusted by 2, the Type 1 error rate was less than $9 \%$. On the other hand, and not surprisingly, the method using no adjustment was consistently the most powerful. Since these methods were using a t-test of $\mathrm{H}_{0}: \beta_{1}=0$, it was not truly clear what the degrees of freedom should be. We used the denominator of the residual variance rounded to the nearest integer. 
Table B-4.2.1. Summary of testing results for the sexond set of simulations. The percent of runs in which the hypothesis $\mathrm{H}_{0}: \beta_{1}=0$ was rejected is shown in the body of the table. The last four columns correspond to the residual variance estimate used, as shown in Equation Bt.2.13. The results are based on 1,000 runs.

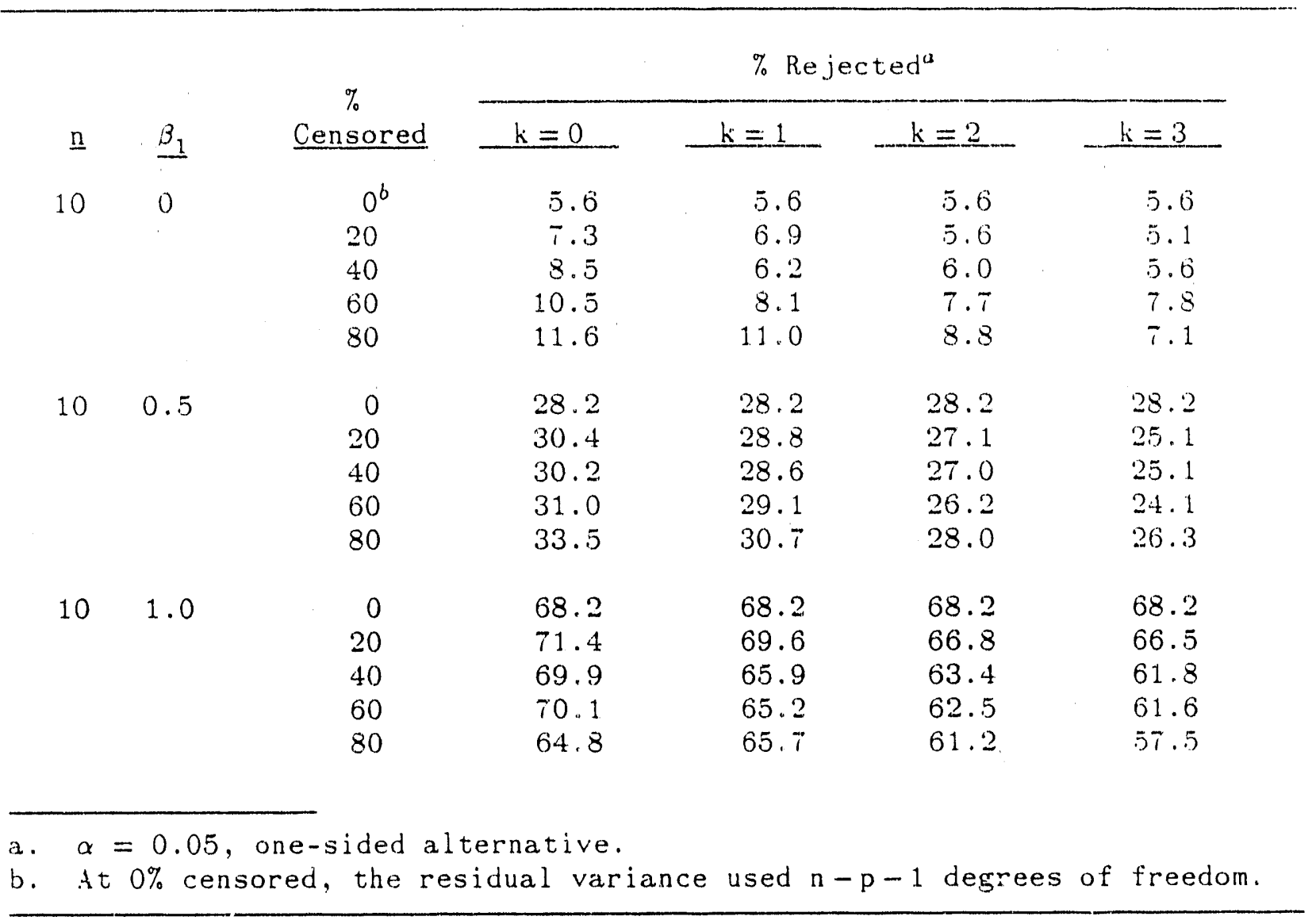


The third set of simulations (which used 5,000 simulations) used only Wolynetz estimate with either no adjustment or an adjustment of 2. The purpose of the third set of simulations was to determine whether the asymptotic test of $\mathrm{H}_{0}: \beta_{1}=0$ performed better than the ad-hoc t-test. The results are presented in Table B-4.2.2. The non-asymptotic results should resemble the corresponding results in Table B-4.2.1, since they are the same methods, but use different random number generator seeds. The asymptotic test was more conservative in that it was less likely to reject the null hypothesis. Its power is sharply reduced at the $60 \%$ censoring level compared to the non-asymptotic test. On the other hand, the asymptotic test did not tend to inflate the $\alpha$-level as does the nonasymptotic test; indeed, the $\alpha$-level falls off at 60 and $80 \%$ censoring in the asymptotic test.

Although not presented in detail here, the method that provided the most unbiased estimates and smallest standard errors for $\mu$ and $\beta_{1}$ was the Wolynetz estimate with no adjustment. This method also provided the best estimate of $\sigma^{2}$ at the higher censoring levels. At low censoring levels, the adjustment of 2 to the denominator appeared to work best at estimating $\sigma^{2}$.

However, at low censoring levels, virtually all the Wolynetz estimates and both the asymptotic and non-asymptotic testing methods work well. It is only at high censoring levels that any differences in performance become apparent. Thus selecting the "best" method will depend upon the objectives of the researcher. Certainly if one is only interested in estimating the model parameters $\mu$ and $\beta_{1}$ it is best to use no adjustment on the residual variance. For testing the hypothesis of no group effect, the recommendations are less clear. If one was most interested in controlling the Type I error rate, it is probably best to use the asymptotic test with no adjustment to the residual variance. If one is more interested in minimizing the Type II error, then the non-asymptotic test should be used. 
Table B-4.2.2. Summary of testing results for the third set of simulations. The percent of runs in which the hypothesis $\mathrm{H}_{0}: \beta_{1}=0$ was rejected is shown in the body of the table. The $k$ value refers to the residual variance adjustment as shown in Equation B4.2-13. Non-asymptotic results are based on a t-test. Asymptotic results are based on a z-test. The results are based on 5,000 runs.

\begin{tabular}{|c|c|c|c|c|c|c|}
\hline \multirow[b]{3}{*}{$\underline{\mathrm{n}}$} & \multirow[b]{3}{*}{$\underline{\beta_{1}}$} & \multirow{3}{*}{$\begin{array}{c}\% \\
\text { Censored }\end{array}$} & \multicolumn{4}{|c|}{$\%$ Rejected ${ }^{a}$} \\
\hline & & & \multicolumn{2}{|c|}{ Non-asymptotic } & \multicolumn{2}{|c|}{ Asymptotic } \\
\hline & & & $k=0$ & $k=2$ & $k=0$ & $k=2$ \\
\hline \multirow[t]{5}{*}{10} & 0 & $0^{b}$ & 5.1 & 5.1 & 5.1 & 5.1 \\
\hline & & 20 & 6.4 & 5.2 & 6.4 & 5.5 \\
\hline & & 40 & 6.9 & 5.8 & 6.2 & 4.9 \\
\hline & & 60 & 8.6 & 6.9 & 5.3 & 3.7 \\
\hline & & 80 & 11.5 & 8.3 & 1.6 & 0.8 \\
\hline \multirow[t]{3}{*}{10} & 0.5 & 0 & 28.1 & 28.1 & 28.1 & 28.1 \\
\hline & & 20 & 31.5 & 27.8 & 32.7 & 29.0 \\
\hline & & 60 & 33.9 & 29.4 & 22.3 & 17.6 \\
\hline \multirow[t]{3}{*}{10} & 1.0 & 0 & 70.3 & 70.3 & 70.3 & 70.3 \\
\hline & & 20 & 73.3 & 69.5 & 74.6 & 71.0 \\
\hline & & 60 & 70.4 & 66.0 & 52.6 & 45.8 \\
\hline \multirow[t]{5}{*}{20} & 0 & 0 & 4.9 & 4.9 & 4.9 & 4.9 \\
\hline & & 20 & 5.6 & 5.1 & 5.6 & 5.1 \\
\hline & & 40 & 6.2 & 5.6 & 5.4 & 5.0 \\
\hline & & 60 & 8.1 & 7.5 & 5.2 & 4.3 \\
\hline & & 80 & 12.2 & 11.2 & 3.4 & 2.3 \\
\hline \multirow[t]{3}{*}{20} & 0.5 & 0 & 46.9 & 46.9 & 46.9 & 46.9 \\
\hline & & 20 & 48.5 & 46.6 & 48.5 & 46.6 \\
\hline & & 60 & 49.5 & 47.5 & 39.1 & 36.3 \\
\hline \multirow[t]{3}{*}{20} & 1.0 & 0 & 92.9 & 92.9 & 92.9 & 92.9 \\
\hline & & 20 & 93.2 & 92.4 & 93.2 & 92.4 \\
\hline & & 60 & 90.2 & 39.3 & 84.5 & 82.9 \\
\hline
\end{tabular}




\section{B-5 LINEAR MODELS}

In a standard linear model, we assume that the observations are independent and normally distributed with a constant variance, and that the means of the observations depend in some linear way on unknown parameters. That is, the observations $Y_{i}$ are normal $\left(\mu_{i}, \sigma^{2}\right)$, where the $\mu_{i}{ }^{\prime}$ s depend on a small number of parameters. In the context of environmental measurements, suppose that the measurements $X_{i}$ have a lognormal $\left(\mu_{i}, \sigma^{2}\right)$ distribution. That is, they all have the same coefficient of variation, and they have different medians $\exp \left(\mu_{i}\right)$. Let $\gamma_{i}=\ln \left(X_{i}\right)$. If the linear-model assumptions relating the $\mu_{i}{ }^{\prime} s$ is correct, then linear-model methods may be applied to analyze a data set of $x_{i}{ }^{\prime}$ s by letting $y_{i}=\ln \left(x_{i}\right)$ and analyzing the $y_{i}{ }^{\prime}$ s.

The detection limit $D L$ for the original $x^{\prime}$ s corresponds to a limit $\ln (D L)$ for the $y^{\prime} s$. Replacement of a censored $x$ by $D L / 2$ means that the corresponding $y$ is replaced by $\ln (D L / 2)=\ln (D L)$ - $\ln 2$. Other replacement techniques for the $x^{\prime}$ s correspond similarly to replacement techniques for the $y^{\prime} s$.

The methods discussed in this section assume that the data are normally distributed, that is, that the log transformation has already been applied to the original data.

\section{B-5.1 Simulations on TWO-Way ANalysis of Variance with Replacements OOR Censored Values}

\section{B-5.1.1 Introduction}

This section discusses the methods and results of two-way analysis of variance (ANOVA) simulations performed on censored data sets, using several replacement techniques for the censored values. Different censoring levels and data distributions are simulated. The approach is patterned after Gilliom and Helsel (1986). Gilliom and Helsel performed extensive simulations that considered 16 distributions, 3 sample sizes, 4 censoring levels, 8 types of estimators, and 4 quantities to be estimated. The quantities to be estimated 
were all point estimates. The simulation described in this manuscript uses some of the same censoring levels and estimators as Gilliom and Helsel. It is different in that the quantities estimated are mean squares for the effects and within-cell error for a two-way ANOVA. Also, the number of distributions, sample sizes, censoring levels, and types of estimators used are more limited in the present study.

\section{B-5.1.2 Simulation Technique}

Distributions. A two-factor fixed-effects model is used in the simulations. Each factor has two levels. Data are randomly generated to reflect different distributions per cell, or treatment combination. Each cell distribution is normal and has variance of one. For this preliminary work, two distributions are used: the null distribution and one designed to produce significant main effects. Table B-5.1.1 shows cell means and coefficients of variation for the simulations performed.

Table B-5.1.1 Treatment combination means and coefficients of variation (in parentheses) used in simulations.

\begin{tabular}{clllll}
\hline Distribution & $a_{1} b_{1}{ }^{a}$ & & $a_{1} b_{2}$ & $a_{2} b_{1}$ & $a_{2} b_{2}$ \\
& 0 & 0 & 0 & 0 & 0 \\
1 & 0 & & $1.5(0.67)$ & $1.5(0.67)$ & $3(0.33)$
\end{tabular}

a. $a_{1} b_{1}$ indicates that level 1 of factor $A$ and level 1 of factor $B$ are applied.

Constant variance is used to meet the assumption of ANOVA. The choices of means serve to vary the on-average significance of main effects and interaction between factors $A$ and $B$ for the sample size used. For the first distribution, the null distribution, the factor effects are all zero. The second distribution has non-zero main effects but no interaction effect. 
Sample size. Simulations are run with 3 and 8 replicates per cell. For each simulation, the same seed is used to generate data sets using the SAS function RANNOR (SAS Institute Inc., 1988a). For the 3-replicate case, 5000 data sets are generated and for 8 replicates, 2000 data sets are generated. programs used to create data sets are found in Section B-5.1.5. The simulations require that at least two uncensored observations remain in each data set following censoring. In addition, at least two uncensored observations are required in each cell for simulations that involve replacement by cell rather than by data set. Because of this requirement, the actual number of data sets used in simulations varies from the original number generated.

\section{Censoring and Replacements.}

Censoring-Type I censoring is performed by determining a cutoff (analogous to a detection limit in environmental data sets) from the overal1, or joint distribution for each simulation. The cutoff $z$ is defined such that

$P(X<Z)=C$

where

$$
\begin{aligned}
& X=\text { random variable for the joint distribution } \\
& c=\text { censoring level. }
\end{aligned}
$$

The censoring is based on the joint distribution because it is more realistic than simply censoring each cell equally. The probabilistic approach is used in preference to percentile censoring based on the data because the latter would represent Type II censoring, not Type I censoring. The censoring levels used correspond to $0 \%, 20 \%$, and $60 \%$ censoring.

Replacements-For the simulations, three types of replacements are used for the censored values: 
- replacement by one-half the cutoff in the lognormal scale, which is replacement by one-half the detection limit in environmental data sets,

- replacement symmetric about one-half the cutoff in the lognormal scale, which is replacement from the distribution that is uniform on the interval with endpoints zero and the detection limit,

- replacement using extrapolation of the regression of the normal data on normal scores, parameters for which are estimated from uncensored data.

Replacement by one-half the cutoff is performed in the following manner:

$$
\begin{aligned}
x & =\ln \left[\exp \left(x_{c}\right) / 2\right] \\
& =x_{c}-\ln (2)
\end{aligned}
$$

where

$x_{c}$ is the cutoff for data generated from normal distributions and

$x$ is the replacement value for data generated from normal distributions that are less than $x_{c}$.

The replacement is performed in this manner because environmental data sets are approximately lognormal in distribution and in practice the replacement would be made in that scale.

The second replacement is also performed in the lognormal scale:

$x_{i j}=\ln \left[\left(y_{c} \times i\right) /\left(n_{c j}+1\right)\right], \quad i=1,2, \ldots n_{c j}$

where 
$y_{c}$ is $\exp$ (the cutoff for data generated from normal distributions),

$x_{i j}$ is the $i$ th replacement value for the $j$ th cell of the data set, and

$n_{c j}$ is the number of censored values in the $j$ th cell.

This type of replacement allows more variability in the replacement values, which may reflect the true data values better than the first technique. The technique is performed by cell.

Replacement using extrapolation of the regression of concentration on normal scores follows that described in Gilliom and Helsel (1986). The technique here is the same as the LR method presented in Section B-3.1.2, except here the observations have already be transformed to normal by taking logs. Normal scores are computed for each uncensored observation using

$z=\Phi^{-1}(r /(n+1))$

where

$\Phi^{-1}$ is the inverse cumulative normal distribution function,

$r$ is the observation rank, and

$n$ is the sample size for the cell.

A least squares regression of observations on normal scores for uncensored data in a cell is extrapolated to estimate censored observations for that ce11. SAS procedures RANK (SAS Institute Inc., 1988b) and REG (SAS Institute Inc., 1988c) are used to perform the ranking and regression, respectively.

In discussion of results, an abbreviation 'hf' (for 'half') is used to represent replacement of censored values by one-half the censoring cutoff. Symmetric replacement about one-half the cutoff is indicated as 'un' because the technique is based on the assumption that the censored values follow a 
uniform distribution. The abbreviation ' $n r^{\prime}$ indicates that normal scores are used in the regression replacement technique.

Simulation statistics. For each simulation, power of mean squares for main and interaction effects and the relative bias of mean squares for main effects, interaction, and within-cell error are computed. Variability in mean squares is expressed in terms of variance, standard error, and relative mean squared error. Errors in power and relative bias are also calculated.

Relative bias is calculated in the following manner:

$$
\left\{\left[(1 / N) \sum_{i=1}^{N} m s_{i}\right]-\text { ems }\right\} / \text { ems }
$$

where

$N$ is the number of data sets with a given censoring and type of replacement,

$\mathrm{ms}_{i}$ is the mean square of the ith data set with a given censoring and type of replacement, and

ems is the mean square that is expected with no censoring.

Formulas for determining ems for two-way ANOVA with fixed effects may be found in Neter and Wasserman (1974, p. 577). For the null distribution, ems $=1$. For the second distribution used in this study and 3 replicates per cell, ems $=7.75$ for main effects and ems $=1$ for interaction and error. With 8 replicates, the main effects ems is 18 for the second distribution.

The error in relative bias is computed

$$
\mathrm{s}_{\mathrm{rns}} /\left[\operatorname{ems} \times\left(N^{1 / 2}\right)\right]
$$


where

$s_{\text {ins }}$ is the sample error of the mean squares for data sets with a given censoring and type of replacement,

and $\mathrm{N}$ and ems are as described above.

The relative mean square error is

N

$\left[(1 / N) \sum\left(m s_{i}-e m s\right)^{2}\right] / e m s^{2}$

$i=1$

This is equal to

$\left[s^{2}+B_{m s}^{2}\right] / e m s^{2}$

where

$s^{2}$ is is the sample variance of the mean squares for data sets with a given censoring and type of replacement,

$B_{m s}$ is the sample bias of the mean squares for data sets with a given censoring and type of replacement, and

ems is as described for relative bias calculations. Expression (B5.1-1) for relative mean square error is used in computations.

Power is computed from the count of the number of data sets that rejected the hypothesis of no main or interaction effects, for a given censoring level and type of replacement.

The simulations are run using SAS procedures MEANS, UNIVARIATE, FORMAT, and TABULATE (SAS Institute Inc., 1988b) and ANOVA (SAS Institute Inc., 1988C). Simulation programs are found in Section B-5.1.5. 
Simulations Performed. Two groups of simulations are performed:

1) Simulations with 3 replicates per cell and replacements by one-half the cutoff and symmetric about one-half the cutoff and

2) simulations with 8 replicates per cell and replacements by one-half the cutoff and regression extrapolation.

The first set of simulations uses the two distributions shown in Table B-5.1.1 and the second set uses the second distribution. Both sets use censoring levels of $20 \%$ and $60 \%$. A11 replacements are done by ce11. (In the case of replacement by one-half the cutoff, replacement by cell within each data set is equivalent to replacement by data set.)

The motivation for the first group of simulations is twofold: 1) environmental data are typically expensive to collect, especially in regulatory settings and 2) the volume of material available for "replication" is limited. (Often true replicates are not possible, only collocated or split samples.) Therefore, a small sample size is used to more realistically simulate practical situations. The requirement of at least two uncensored values in each data set is applied, since as a matter of practicality, data sets with fewer uncensored values would probably not be subjected to analysis. As a result of this requirement, a larger number of data sets contribute co the statistics at lower censoring levels compared to higher censoring levels. The number of data sets is also dependent on the joint distribution of the data generated.

The second group of simulations is performed primarily to provide a basis for evaluating the regression method in the two-way ANOVA application. A larger sample size is used in these simulations for the following reasons:

1) reasonable use of the regression method requires replacement by cel1 and 
2) the regression method requires two uncensored values for estimation.

Therefore, the requirement of two or more uncensored values in each cell of a data set is applied to the simulations. To meet this requirement and in order to have a large number of acceptable data sets generated in a reasonable amount of computing time, a larger sample size is required.

\section{B-5.1.3 Simulation Results}

Power. Table B-5.1.2 shows the power results for simulations performed using 3 replicates per cell. The replacement techniques examined are one-half the censoring cutoff (indicated as ' $h f^{\prime}$ ) and symrietric about one-half the cutoff (indicated as 'un'). The results show that the power decreases with an increase in censoring frum $20 \%$ to $60 \%$ for a 11 treatment effects. The trend is more evident with un replacements of censored values. In fact, at $60 \%$ censoring of the null distribution, confidence intervals for the power using the un replacement do not include the true value $(0.05)$ or overlap with confidence intervals for simulations with no censoring. That is, from the $A$ column of the table, $0.0316+2 \times 0.0025$ is well below 0.05 , and even below $0.0486-2 \times 0.0030$; similar results are seen for the $B$ and $A * B$ columns. The hf replacement method also performs poorly in terms of power for the second distribution with $60 \%$ censoring.

One exception to the above exists: the interaction term for the second distribution with $60 \%$ censoring has anomalously high power, which in this instance should ideally be much lower. The tendency would be to reject the hypothesis of no interaction more than expected, which is undesirable.

For the second set of simulations, the replacement techniques examined are $h f$ and extrapolation of the regression of uncensored concentration on normal scores (indicated as ' $n r^{\prime}$ ). The results, shown in Table B-5.1.3, indicate that the hf method has better power, al though not appreciably better power, than the $\mathrm{nr}$ method for the main effects. However, the hf method is 
clearly preferable in testing for significant interaction compared to the nr method. 
Table B-5.1.2. Power results for simulations performed using 3 replicates per cel1. DISTN = distribution (defined in Table B-5.1.1), CENSOR = censoring level, TYPE = type of replacement of censored values (see text), $N$ = number of data sets, SOURCF_ = sources of variation for the two-way setting with factors $A$ and $B^{-}$, POWER ${ }^{-}=$power (or Type I error as appropriate), STDEDrOR = error for power based on binomial distribution.

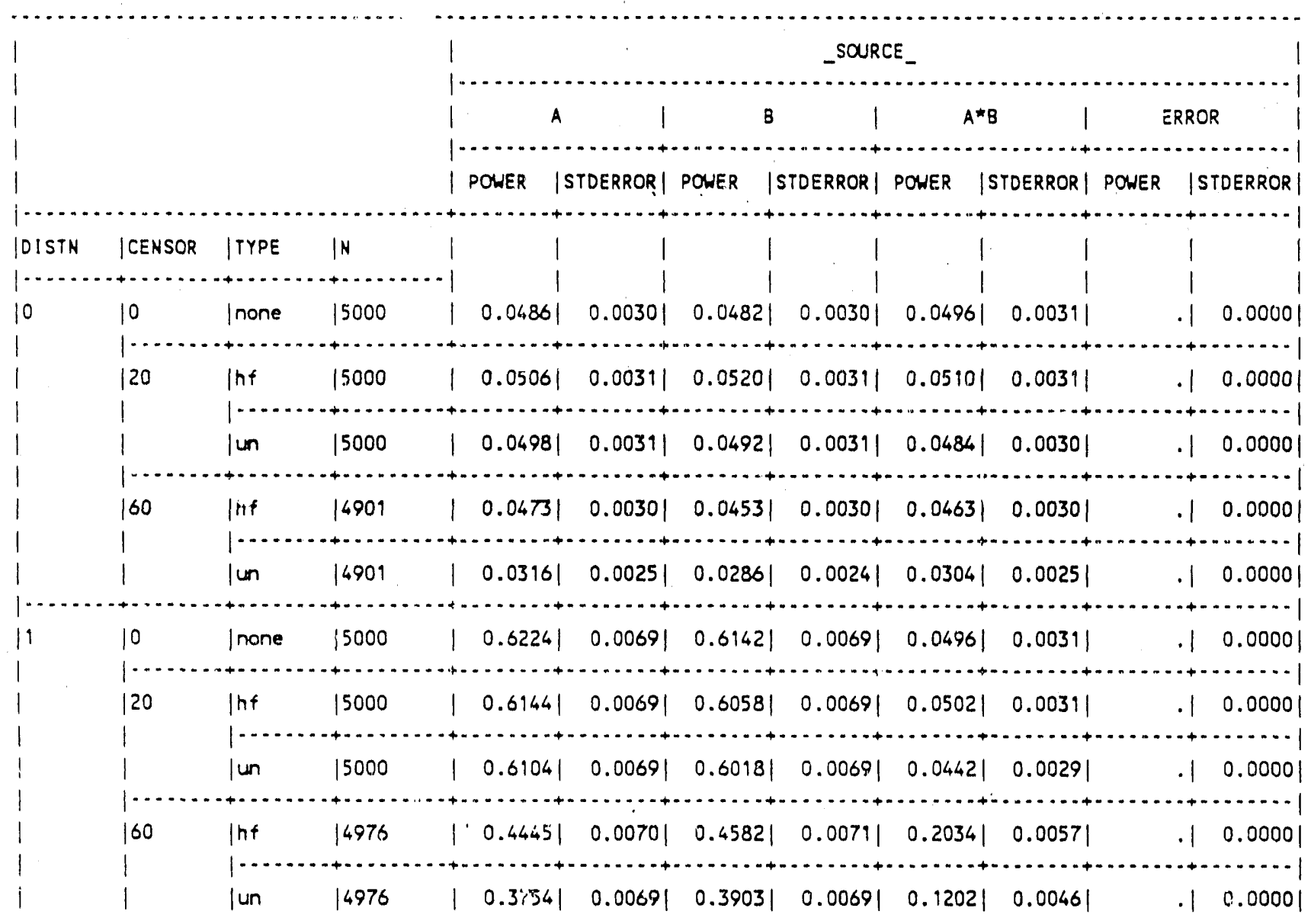


Table B-5.1.3. Power results for simulations performed using 8 replicates per cel1. DISTN = distribution (defined in Table B-5.1.1), CENSOF = censoring level, TYPE = type of replacement of censored values (see text), $N$ = number of data sets, SUURCE = sources of variation for the two-way setting with factors $A$ and $B^{-}$, POWER $=$power (or Type I error as appropriate), STOERROR = error for power based on bincmial distribution.

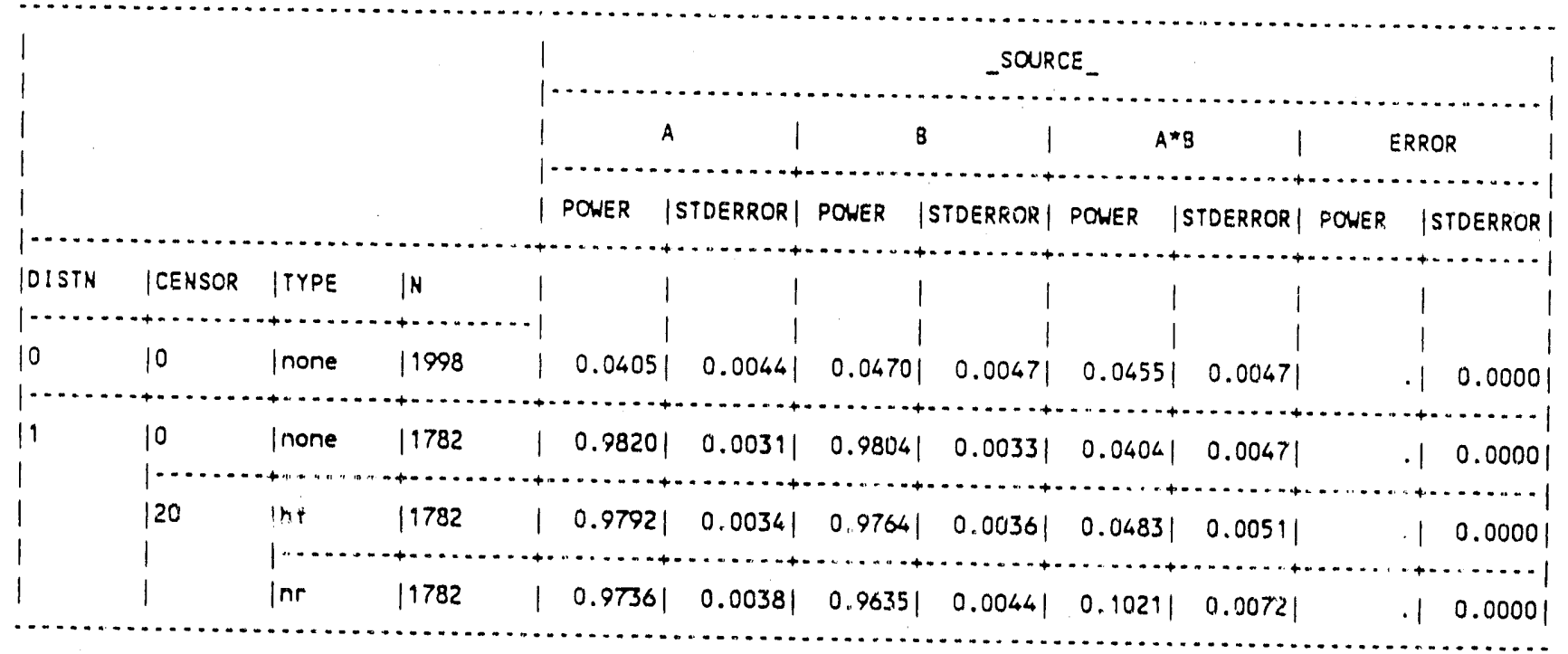


Relative Bias. Table B-5.1.4 shows relative bias for the simulations with 3 replicates per cell and using replacement techniques hf and un. The results show that the absolute value of the bias at the $60 \%$ censoring level is larger than at the $20 \%$ censoring level. The trend is more pronounced with distribution 1 except for the interaction mean square relative bias with $60 \%$ censoring. The reason for the exception is unknown.

There are no differences in relative bias between all combinations of censoring levels and replacement types. For $20 \%$ censoring of the null distribution, replacement using the hf technique is preferable, as the relative bias is less for hf than the un type replacement. However, for $60 \%$ censoring, un has lower (in absolute value) relative bias. The preferred replacement technique for the second distribution based on relative bias is un for both censoring levels.

Distinct differences exist between relative bias of mean squares at the $60 \%$ censoring level and other results. This indicates that, for the most part, use of the replacement techniques hf and un results in severely biased mean squares in situations where $60 \%$ of the measurements are censored, irrespective of the underlying distribution. As for power results, however, the interaction term for the second distribution at $60 \%$ censoring is an anomaly. The relative bias is not extreme as for other sources of variation. Even so, a test for significant interactions would probably be inaccurate because the error mean square is severely biased. The power results bear this out.

Table B-5.1.5 gives the results for the second set of simulations. The relative bias of the interaction mean squares is much better when the hf method is sed rather than the $\mathrm{nr}$ inethod. In fact, the mean square is not different from the case of no censoring. It is interesting to note that the relative biases of mean squares using the $\mathrm{nr}$ method are similar to that for no censoring for the other sources of variation. 
Table B-5.1.4. Relative bias of mean squares for simulations performed using 3 replicates per cell. DISTN = distribution (defined in Table B-5.1.1), CENSOR = censoring level, TYPE = type of replacement of censored values (see text), $N=$ number of data sets, SOURCE = sources of variation for the two-way setting with factors $A$ and $\vec{B}$, RELBIAS $=$ relative bias of mean squares, RBERROR = standard error of relative bias.

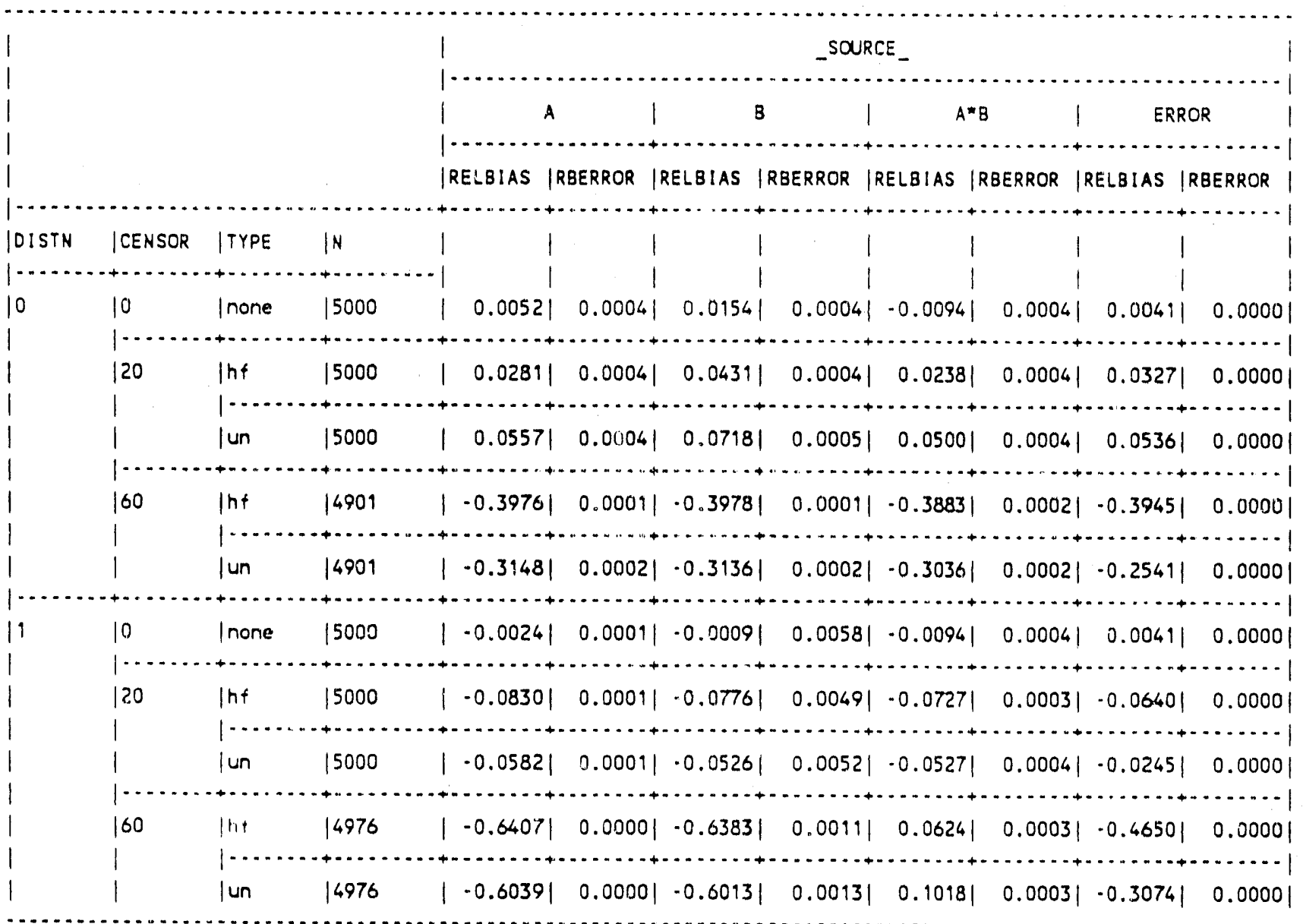


Table B-5.1.5. Relative bias of mean squares for simulations performed using 8 replicates per cell. DISTN = distribution (defined in Table B-5.1.1), CENSOR = censoring level, TYPE = type of replacement of censored values (see text), $N=$ number of data sets, SOURCE = sources of variation for the two-way setting with factors $A$ and $\bar{B}$, RELBIASS = relative bias of mean squares, $R B E R R O R=$ standard error of relative bias.

SAS

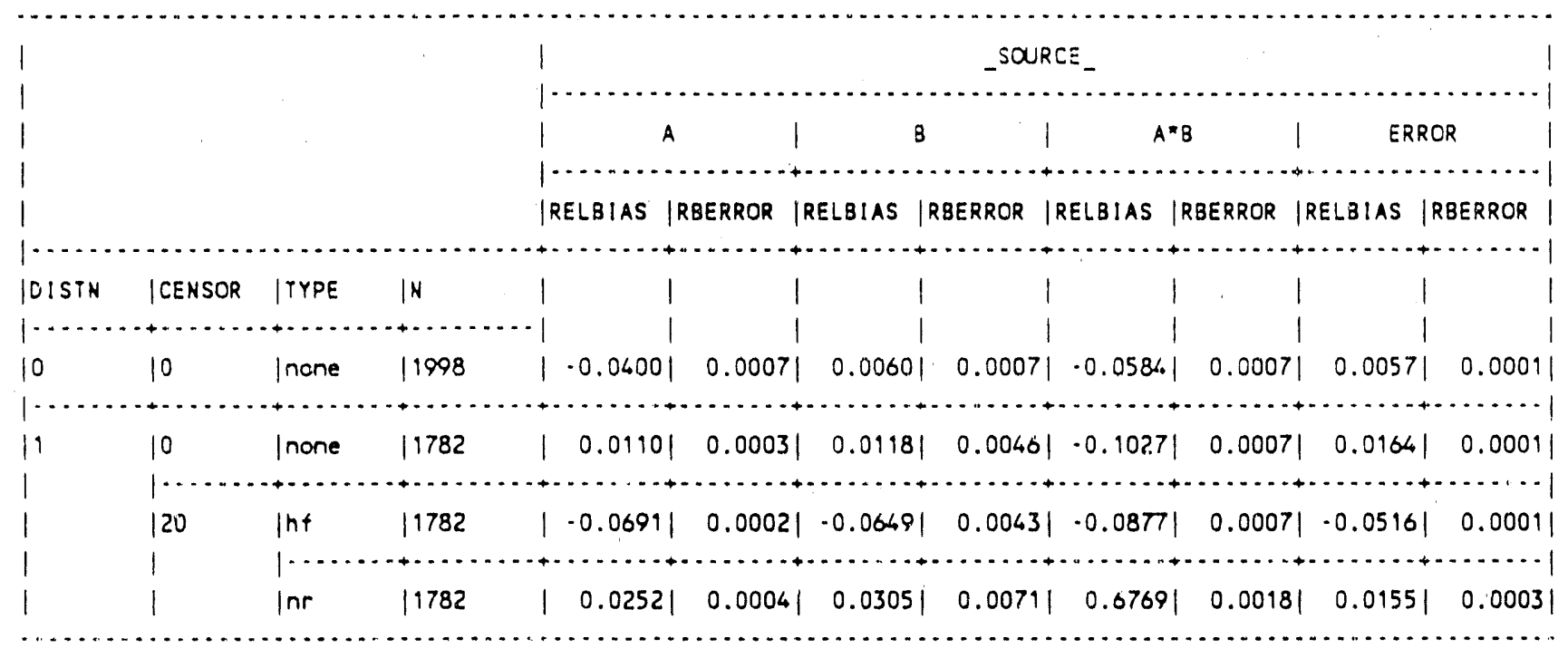


Variance of Mean Squares. Table B-5.1.6 gives the standard error and variance of mean squares for simulations performed using 3 replicates. A similar pattorn as that described for relative bias is observed, with values for $60 \%$ censoring being distinctly different from others. The variance of mean squares is probably lower for $60 \%$ censoring because more values are being replaced with the same or similar values. The variance of hf in general is less than the variance of the un technique for the same reason. The variance of mean squares for main effects for the second distribution is quite high compared to other values. Table B-5.1.7 shows similar results for the second set of simulations.

Relative mean squared error for the mean squares for simulation sets 1) and 2) are given in Tables B-5.1.8 and B-5.1.9, respectively. The estimates are dominated by the variance of mean squares and, for main effects for the second distribution, by the ems factor.

\section{B-5.1.4 Conclusions}

The simulation results lend themselves to somewhat different interpretations, depending on which performance statistic(s) is examined. Because one of the replacement techniques examined inherently reduces variability, variance performance measures may not be reflective of superior performance. A similar problem exists with relative bias, which forms the basis of variance calculations. Power, however, is a measure that reflects the ability of the analysis to make correct assertions concerning the data. Because of this, power is considered the best performance indicator in this study.

Based on power results, hf is the preferred replacement method over the un method for a two-way ANOVA setting. However, with a higher censoring level $(60 \%)$, even the hf method does not perform well for the non-null distribution. Relative bias is also great at the $60 \%$ censoring level. Preliminary work indicates that the hf method is also preferable to the $\mathrm{nr}$ method. 


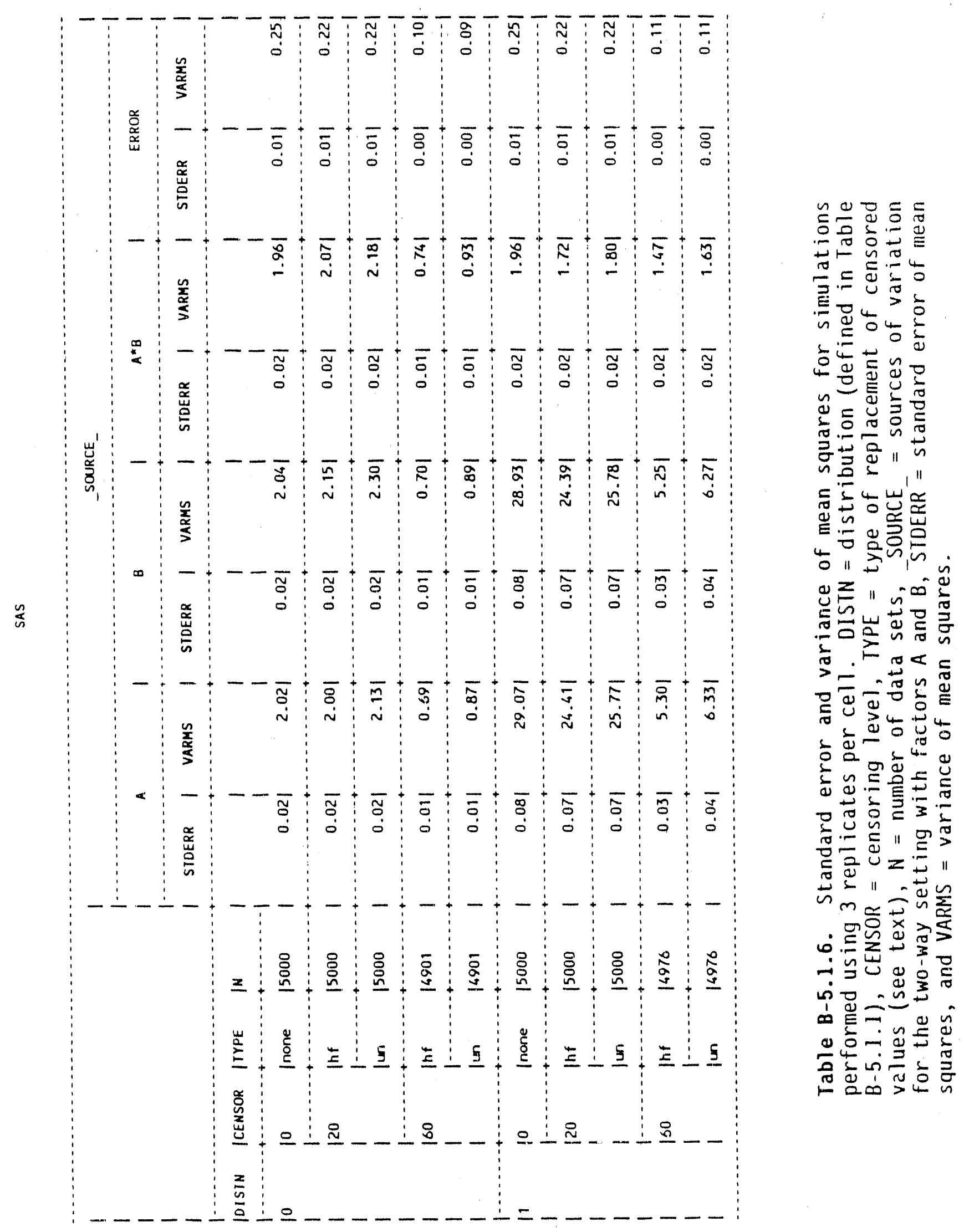




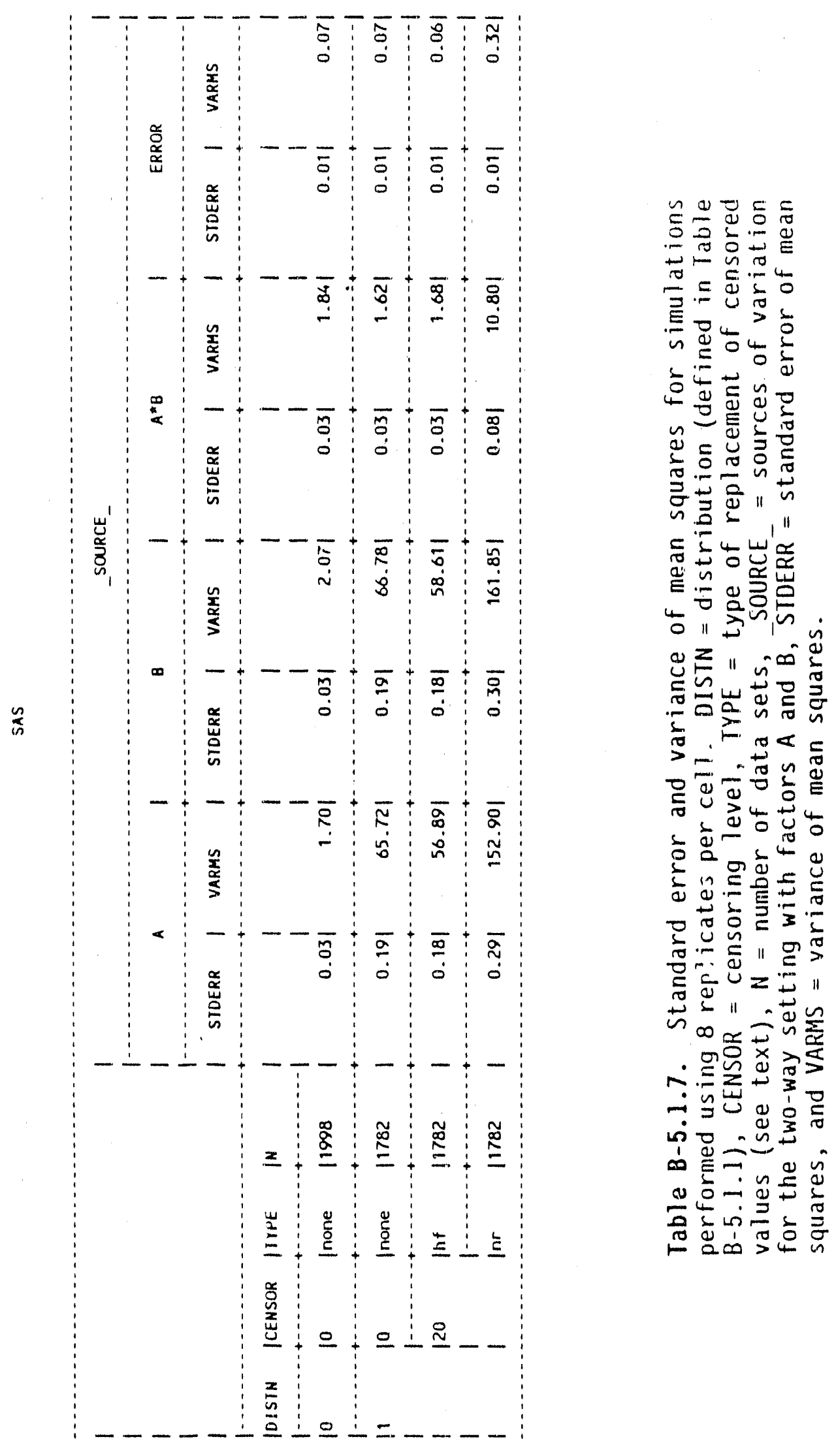


Table B-5.1.8. Relative mean squared error for simulations performed using 3 replicates per cell. DISTN = distribution (defined in Table B-5.1.1), CENSOR = censoring level, TYPE = type of replacement of censored values (see text), $N$ = number of data sets, SOURCE = sources of variation for the two-way setting with factors $A$ and $\bar{B}, R E L M \bar{S} E=$ relative mean squared error of mean squares.

SAS

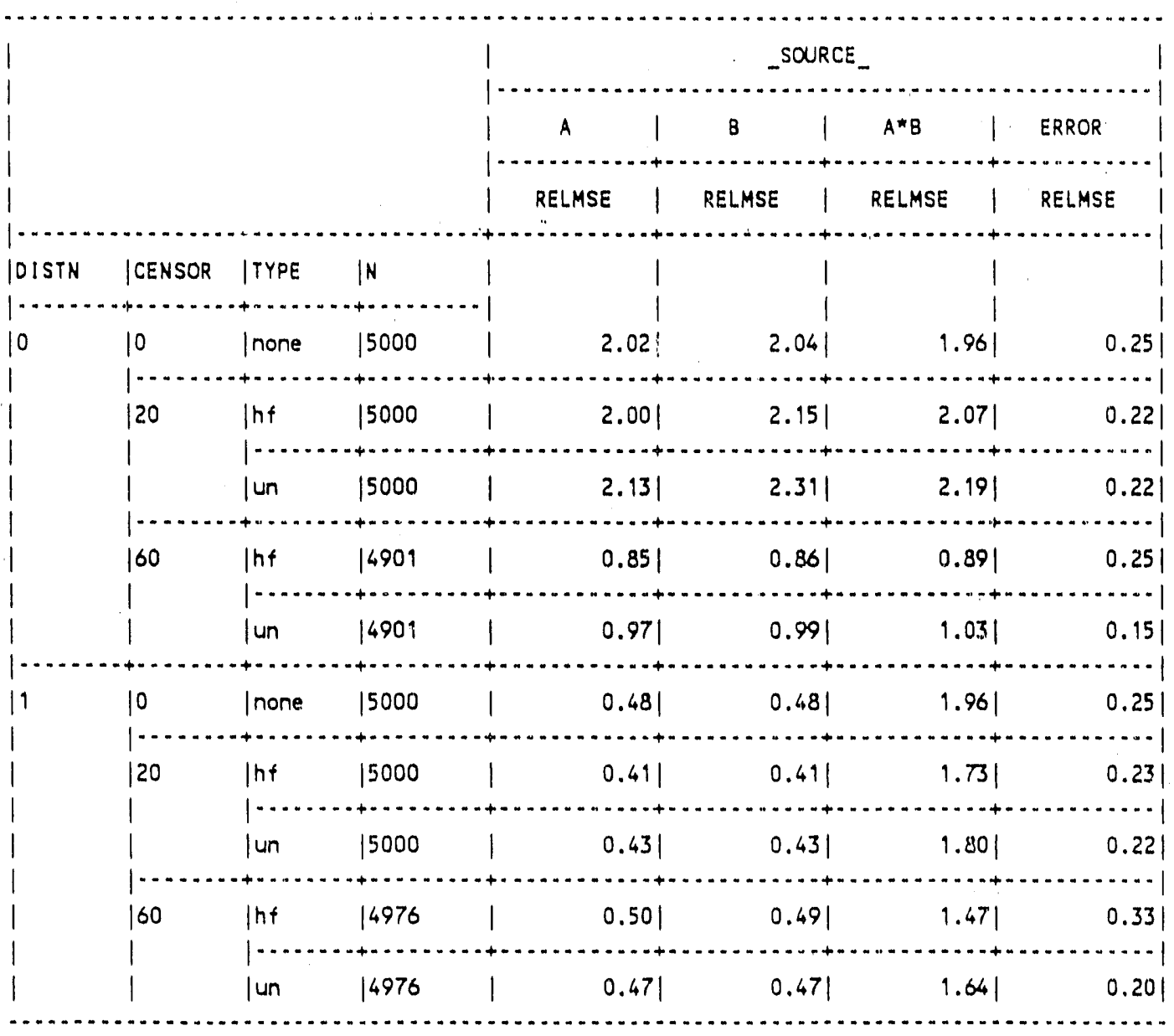


Table B-5.1.9. Relative mean squared error for simulations performed using 8 replicates per cell. DISTN = distribution (defined in Table B-5.1.1). CENSOR = censoring level, TYPE = type of replacement of censored values (see text), $N=$ number of data sets, SOURCE = sources of variation for the two-way setting with factors $A$ and $\bar{B}$, RELMSE = relative mean squared error of mean squares.

SAS

\begin{tabular}{|c|c|c|c|c|c|c|c|c|}
\hline \multicolumn{9}{|l|}{1} \\
\hline & & & & 1 & 1 & 8 & $A * 3$ & ERROR \\
\hline & & & & $1 \cdot$ & $\cdots \cdots+\cdots+\cdots$ & …… & $\cdots \cdots+\cdots+$ & $\cdots \ldots \ldots$ \\
\hline 1 & & & & 1 & RELMSE I & RELMSE $\quad \mid$ & RELHSE I & RELMSE \\
\hline & & & & + & 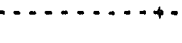 & -+- & $\cdots$ & $\cdots \cdots$ \\
\hline DOISTN & |CENSOR & ITYPE & H & 1 & 1 & 1 & 1 & \\
\hline$|\cdots \ldots|$ & $-+\ldots \ldots$ & $\cdots \cdots+$ & 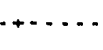 & -1 & 1 & 1 & 1 & \\
\hline 10 & 10 & Inone & 11998 & 1 & 1.701 & 2.071 & 1.841 & $0.07 \mid$ \\
\hline $1 \cdots$ & $++\cdots$ & $+\cdots \cdots$ & $+\cdots$ & ++ & $\ldots \ldots+\ldots+$ & $\ldots \ldots \ldots+$ & $\cdots \cdots+\cdots+\cdots$ & $\cdots \cdots \cdots+$ \\
\hline $\mid 1$ & 10 & Inone & 1782 & I & 0.201 & 0.211 & 1.641 & 0.071 \\
\hline 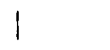 & $|\cdots \ldots|$ & $-+\cdots \cdot$ & $-+\cdots \cdot$ & -+ & $\ldots \ldots \ldots+\cdots$ & …......... & $\ldots \ldots \ldots+$ & $\cdots \ldots \ldots \ldots$ \\
\hline i & 120 & lhf & 11782 & 1 & 0.181 & 0.191 & 1.681 & 0.071 \\
\hline . & 1 & $1 \cdots$ & $+\cdots \cdots$ & 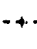 & $\ldots \ldots \ldots+\cdots+$ & $\cdots \cdots \cdots+\cdots$ & $\cdots \cdots+\cdots+$ & $\cdots \cdots \cdots+1$ \\
\hline 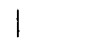 & 1 & Inr & 11782 & I & 0.471 & 0.501 & 11.261 & 0.32 \\
\hline
\end{tabular}


Future work would attempt to identify censoring level/distributional setting at which performance of hf becomes unacceptable. Other work would address performance in the presence of interaction. Future work should also attempt to clarify inconsistencies observed in this study and to further evaluate the nr method.

\section{B-5.1.5 Programs for Data Generation and ANOVA Simulation}

The following SAS programs were used to generate the data sets and produce the results tabulated above.

PROGRAM FOR DATA GENERATION AND ANOVA SIMULATION

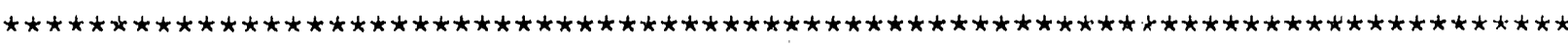

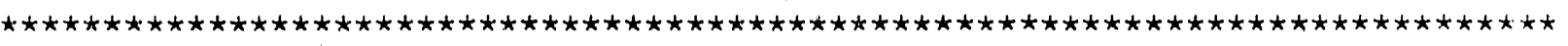
Variables changed and sections modified for simulation runs are indicated in bold italicized print. Comments are preceded with asterisks.

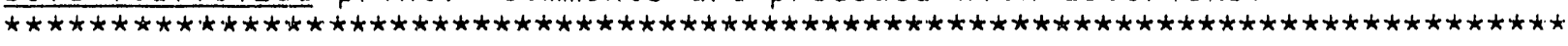

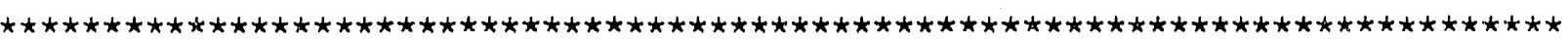

* Generate data:

$k 2 \times 2$ data sets with $r$ reps

cell means $t, u$, and $v$

$x n n$ is censoring cutoff value and represents the $n$th

percentile such that sum censored from $N(0,1), N(t, 1)$,

$N(u, 1)$, and $N(v, 1)$ is $n / 100$

screen data sets using 'drop2.sas' (by cell)

or 'drop.sas' (by data set)

;

libname erdp 'c: \ltderdp \test';

data gendat;

$r=8 ; \quad k=2000 ;$ dat $=1 ; \quad f 7 a g=1 ; \%$ let seed $=160589$;

$t=1.5 ; \quad u=3 ; v=1.5 ;$

do $i=1$ to $k^{\star} r$

$y l=\operatorname{rannor}(\&$ seed $)$;

yz=t+rannor (\&seed);

y3 $=u+r a n n o r(\&$ seed);

y $4=v+r a n n o r(\&$ seed) ;

if $i / r<=r^{*}$ dat then $d a t=f 1 a g$;

if $\bmod (i, r)=0$ then $f 1 \mathrm{ag}=\mathrm{flag}+1$;

output; end;

drop $k$ flag $t u v i r$; 
data one; set gendat;

keep y we11 depth dat;

wel1 $=$ ' $\mathrm{a}^{\prime} ; \quad \operatorname{depth}=5 ; \quad \mathrm{y}=\mathrm{yl}$;

data two; set gendat;

keep y well depth dat;

we $11={ }^{\prime} \mathrm{a}^{\prime} ; \quad \operatorname{depth}=10 ; \quad \mathrm{y}=\mathrm{y} 2$;

data three; set gendat;

keep y well depth dat;

well='b'; $\operatorname{depth=10;y=y3;}$

data four; set gendat;

keep y well depth dat;

wel1 $=$ ' $b^{\prime} ; \quad \operatorname{depth}=5 ; \quad y=y 4$;

data gendat; set one two three four;

$\underline{x n n}=0.2533$;

$\%$ include 'cellset2.sas';

\%include 'clear.sas';

*eliminate data sets with $<2$ uncensored values in each cell;

proc sort data=gendat; by dat cell;

proc means data=gendat noprint $n$; var $y$; by dat; output out $=a 11 \mathrm{cnt} n=a 11 \mathrm{cnt}$;

data drop; set gendat; if $y>=x n n$ then $f 1 a g=l$; el se $f l a g=0$;

\%include 'drop2.sas';

data gendat;merge gendat build; if dat ne drop;

drop _TYPE__FREQ_ drop;

data build; set clear;

*Perform censored value replacement:

replacement section changes according to replacement technique simulated;

data gendat; set gendat; $c u t=x n n ;$ drop $x n n$;

\%include 'nr.sas':

data gendat; set built one:

drop nscore cuti

data one; set clear:

data builtiset clear;

*Produce anova statistics:

output file names and ems change according to simulation;

proc scrt data=gendat;

by dat; 


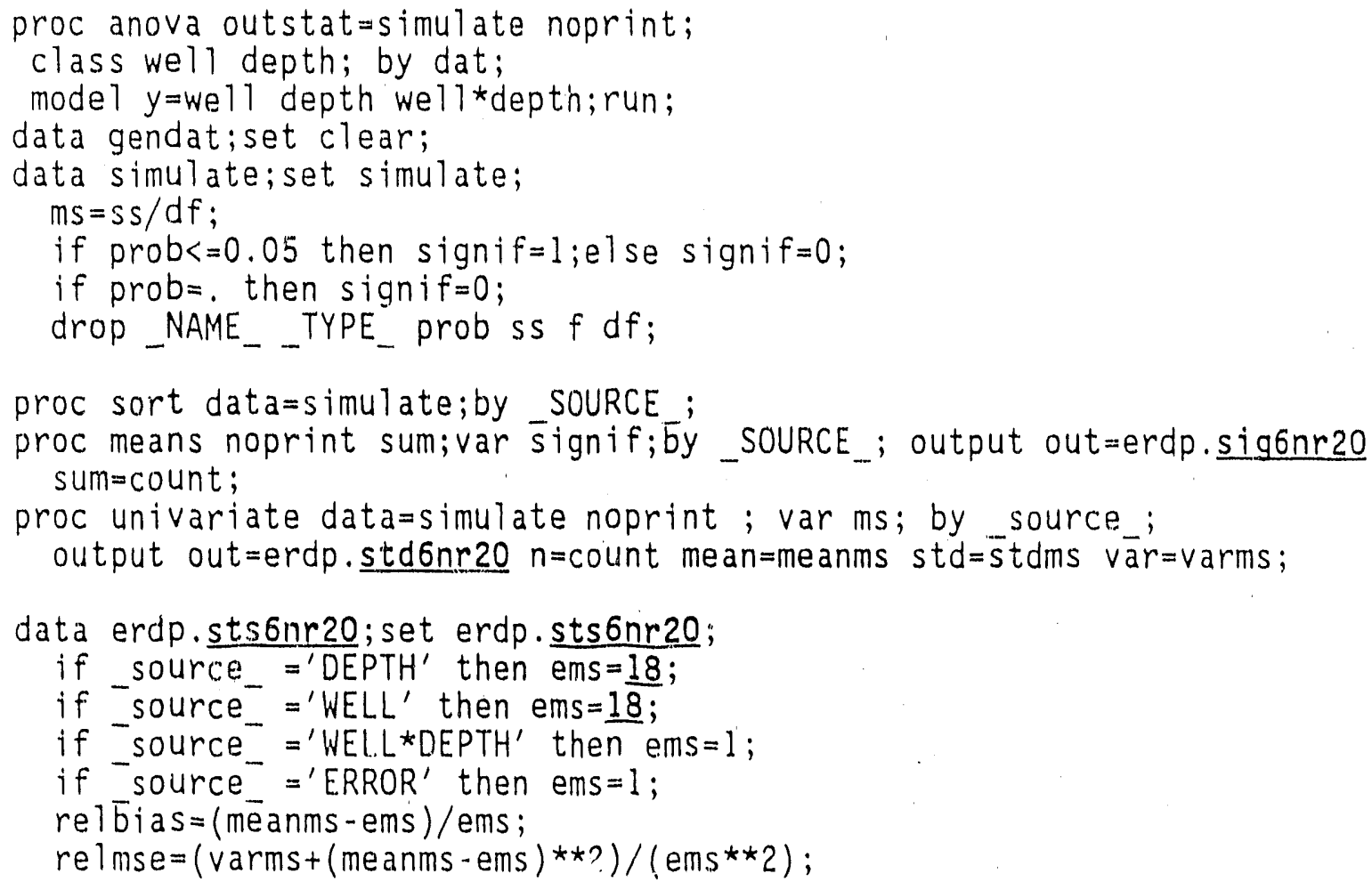




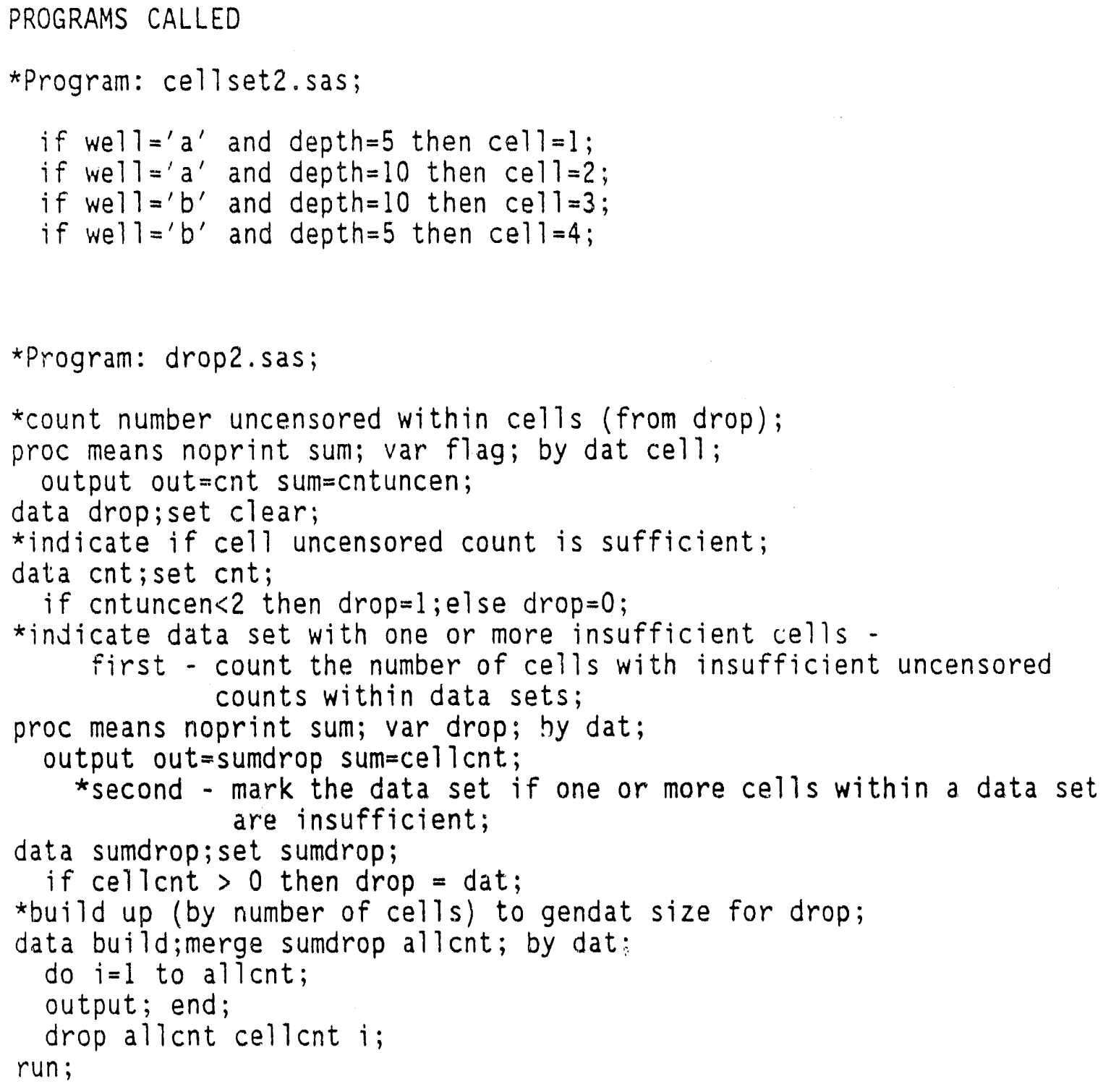

^Program: drop2.sas;

* count number uncensored within cells (from drop);

*Program: drop.sas;

proc means noprint $n$; var $y$; by dat; output out=cnt $n=$ cntuncen;

data build;merge cnt allcnt; by dat;

do $i=1$ to allent;

if cntuncen<2 then $d r o p=d a t ; e l s e d r o p=$. ;

output; end;

drop allent cnturicen $i$; 


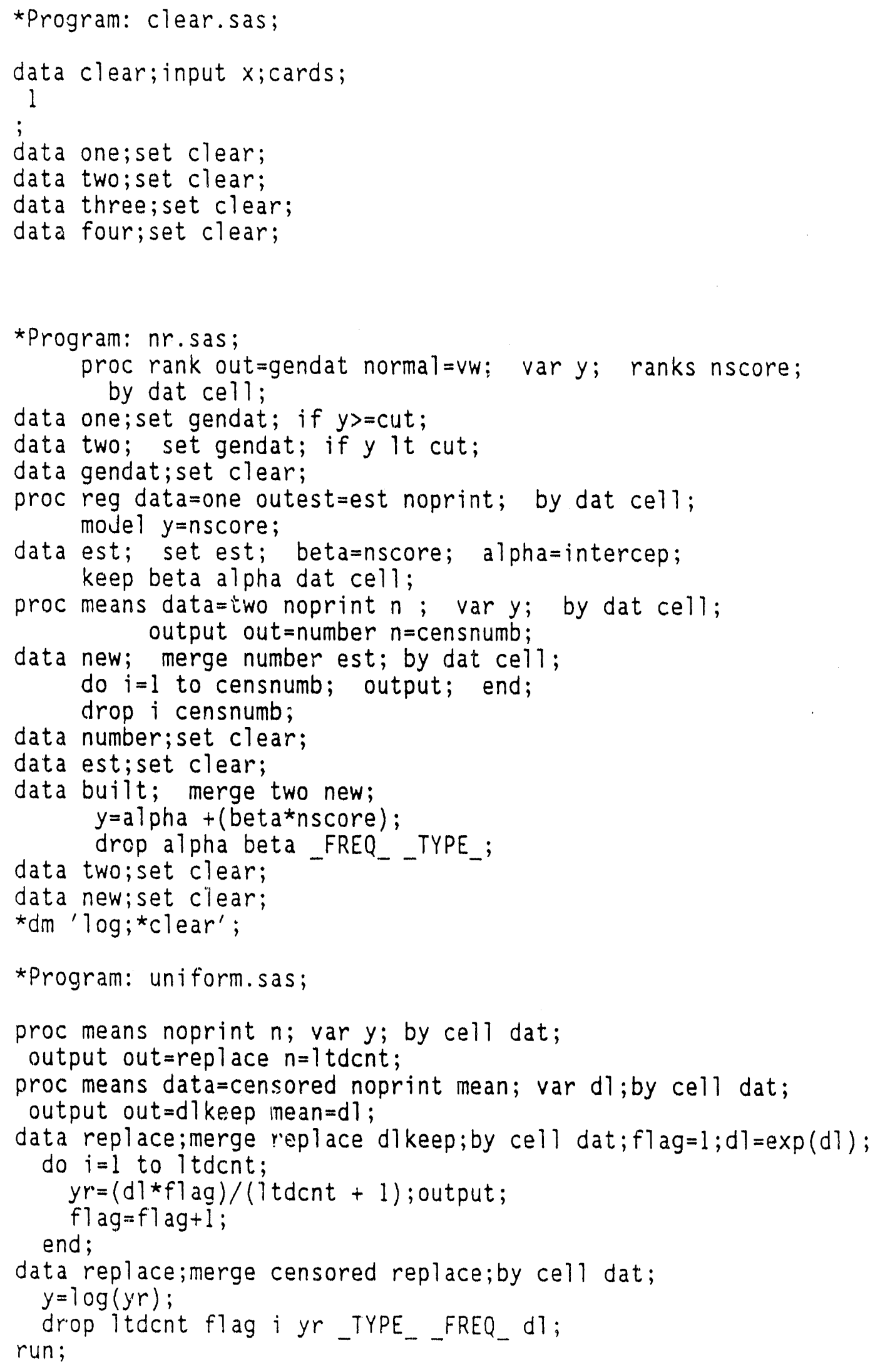




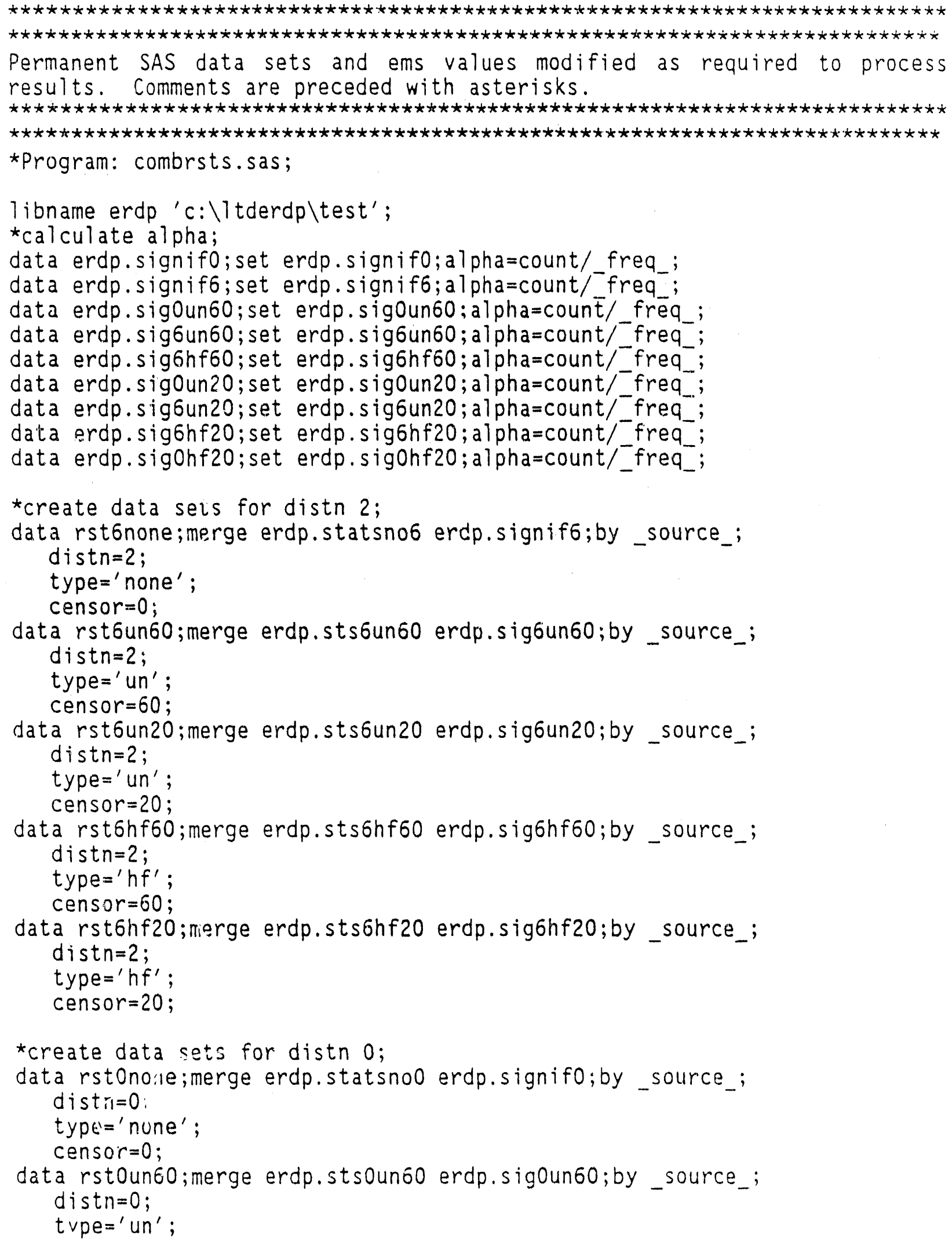


censor $=60$;

data rstoun20;merge erdp.stsoun20 erdp.sigoun20; by _source_;

distn=0;

type $={ }^{\prime}$ un $^{\prime}$;

censor $=20$;

data rstohf60;merge erdp.sts0hf60 erdp.sig0hf60; by _source_;

distn=0;

* combine all data sets, calculate additional statistics, and set up formats; data erdp.a11; set

rst6none

rst6un60

rst6un20

rst6hf60

rst6hf20

rstonone

rstoun60

rstoun20

rstohf60

rstohf20

;

stderr=stdms/sqrt (_freq_);

stdalpha=sqrt (alphä $*(1-\bar{a}$ ?pha)/_freq_);

if source $=$ 'ERROR' then alpha $=\overline{-}$;

if source $^{-}={ }^{\prime}$ ERROR' then source $={ }^{\prime} Z^{\prime}$;

if _source_- $=$ 'WELL*DEPTH' Then_source_ $={ }^{\prime} X^{\prime}$;

if distn=2 and source $=$ 'WELL' then ems $=7.75$;

if distn=2 and source $={ }^{\prime}$ DEPTH' then ems $=7.75$;

else ems=1;

rberror=stderr $/(($ sart $($ freq_ $)) *$ ems $)$;

rbupc $i=$ relbias + (2*rberror $)$;

rblowci=relbias - (2*rberror);

options nonumber nodate $p s=44 \quad 1 s=155$;

proc sort; by _source_;

proc format;

value \$_source 'DEPTH' ${ }^{\prime} A$ '

$$
\begin{aligned}
& \text { 'WELL } L^{\prime}={ }^{\prime} B^{\prime} \\
& Z^{\prime}={ }^{\prime} E R R O R^{\prime} \\
& X^{\prime}={ }^{*} A^{*} B^{\prime} ;
\end{aligned}
$$

*tabulate results;

proc tabulate data=erdp.a17;

class censor type freq__ source distn;

var alpha relbias stder $\bar{r}$ varms sẼdalpha rberror rbupci rblowci ralmse; 


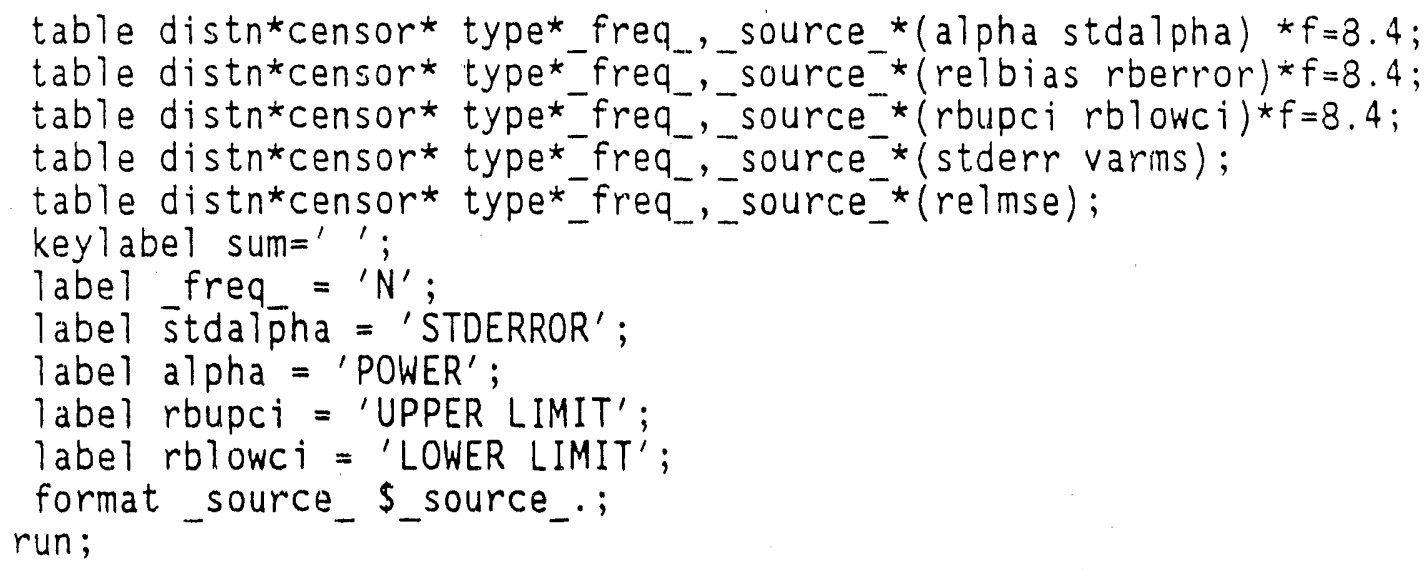




\section{REFERENCES}

J. Aitchison and J. A. C. Brown, The Lognormal Distribution with Special Reference to Its Use in Economics, Cambridge: Cambridge University Press, 1957 .

M. Aitkin, "A Note on the Regression Analysis of Censored Data," Technometrics, 23, 1981, pp. 161-163.

M. M. Bernarie, "The Validity of the Log-normal Distribution of Pollutant Concentrations," Paper SU-18D, Proceedings of the 2nd International Clean Air Conference, (CE-Trans--7589), 1971.

Larry G. Blackwood, "Estimating Population Means and Standard Deviations from Censored Samples: An Evaluation of Gupta's Method," unreleased report, EG\&G Idaho Inc.: Idaho Falls, ID, 1989.

Larry G. Blackwood, The Lognormal Distribution, Environmental Data, and Radiological Monitoring, EGG-SARE-9243, September 1990.

L. G. Blackwood, "The Quality of Mean and Variance Estimates for Normal and Lognormal Data when the Underlying Distribution is Misspecified, "Journal of Chemometrics (to appear, 1991).

Timothy A. Cohn, "Adjusted Maximum Likelihood Estimation of the Moments of Lognormal Populations from Type 1 Censored Samples," U. S. Geo7. Survey Open File Rep. 88-350, 1988.

E. L. Crow, "Applications in Atmospheric Sciences," Chapter 13 in Lognormal Distributions: Theory and Applications, (E. L. Crow and K. Shimizu, eds.), New York: Marcel Dekker, Inc., 1988.

E. L. Crow and K. Shimizu (eds.), Lognormal Distributions: Theory and Applications, New York: Marcel Dekker, Inc, 1988.

A. P. Dempster, N. M. Laird, and D. B. Rubin, "Maximum Likelihood from Incomplete Data via the EM Algorithm," and discussion, Journal of the Royal Statistical Society, Series B, 39, 1977, pp. 1-38.

E. Dennis and G. P. Patil, "Applications in Ecology, "Chapter 12 in Lognormal Distributions: Theory and Applications, (E. L. Crow and K. Shimizu, eds.), New York: Marcel Dekker, Inc., 1988.

L. L. Eberhardt and R. 0. Gilbert, Gamma and lognormal distributions as models in studying food-chain kinetics, Battelle, Pacific Northwest Laboratories, BNWL-1747, 1973.

L. L. Eberhardt and R. 0. Gilbert, "Statistics and Sampling in Transuranic Studies," in Transuranic Elements in the Environment (W. C. Hanson, ed.), DOE/TIC-22800 NTIS, 1980, pp. 173-186. 
P. G. Georgopoulos and J. H. Seinfeld, "Statistical Distributions of Air" Quality Concentrations," Environmental Science and Technology, 16, 1982, 401$416 \mathrm{~A}$.

R. 0. Gilbert, Statistical Methods for Environmental Pollution Monitoring, New York: Van Nostrand Reinhold Co., 1987.

Robert J. Gilliom and Dennis R. Helsel, "Estimation of Distributional Parameters for Censored Trace Level Water Quality Data: 1. Estimation Techniques," Water Resources Research, 22, 1986, pp. 147-155.

A. K. Gupta, "Estimation of the Mean and Standard Deviation of a Normal Population from a Censored Sample," Biometrika, 39, 1952, pp. 260-273.

Myles Hollander and Douglas A. Wolfe, Nonparametric Statistical Methods, New lork: John Wiley \& Sons, 1973.

T. R. Johnson and M. J. Symons, "Extreme Values of Weibull and Lognormial Distributions Fitted to Ambient Air Quality Data," presented at the 73rd Annual Meeting of the Air Pollution Control Association, Montreal, Quebec, 1980 .

William J. Kennedy, Jr., and James E. Gentle, Statistical Computing, New York: Marcel Dekker, Inc., 1980.

G. S. Koch and R. F. Link, Statistical Analysis of Geological Data, New York: Dover, 1981.

S. P. Millard and S. J. Deveral, "Nonparametric Statistical Methods for Comparing Two Sites Based on Data with Multiple Nondetect Limits," Water Resources Research, 24, 12, 1988, pp. 2087-2098.

John Neter and William Wasserman, 1974, Applied Linear Statistical Models, Homewood, IL: Richard D. Irwin, Inc., $842 p$.

A. E. Sarhan and B. G. Greenberg, Contributions to Order Statistics, New York: John Wiley and Sons, 1962.

SAS Institute Inc., SAS Language Guide for Personal Computers, Release 6.03 Edition, Cary, NC: SAS Institute Inc., 1988a.

SAS Institute Inc., SAS Procedures Guide, Release 6.03 Edition, Cary, NC: SAS Institute Inc., 1988b.

SAS Insiitute Inc., SAS/STAT User's Guide, Release 6.03 Edition, Cary, NC: SAS Institute Inc., 1988c.

J. Schmee and G. J. Hahn, "A Simple Method for Regression Analysis with Censored Data," Technometrics, 21, 4, 1979. pp. 417-432. 
K. Shimizu, K., "Point Estimation," Chapter 2. in Lognormal Disiributions: Theory and Applications, (E. L. Crow and K. Shimizu, eds.), New York: Marcel Dekker, Inc., 1988.

K. Shimizu and E. L. Crow, "History, Genesis, and Properties, "Chapter 1 in Lognormal Distributions: Theory and Applications, (E. L. Crow and K. Shimizu, eds.), New York: Marcel Dekker, Inc., 1988.

D. R. Speer and D. A. Waite, Statistical Distributions as Applied to Environmental Surveillance Data, Battelle, Pacific Northwest Laboratories, BNWL-SA-5482, 1975.

R. G. D. Steel and J. H. Torrie, Principles and Procedures of Statistics, Second Edition, New York: McGraw-Hi11, 1980.

M. S. Wolynetz, "Algorithm AS 138. Maximum Likelihood Estimation from Confined and Censored Normal Data," Applied Statistics, 28, 1979a, pp. 185195.

M. S. Wolynetz, "Algorithm AS 139. Maximum Likelihood Estimation in a Linear Model from Confined and Censored Normal Data," Applied Statistics, 28. 1979b, pp. 195-214. 

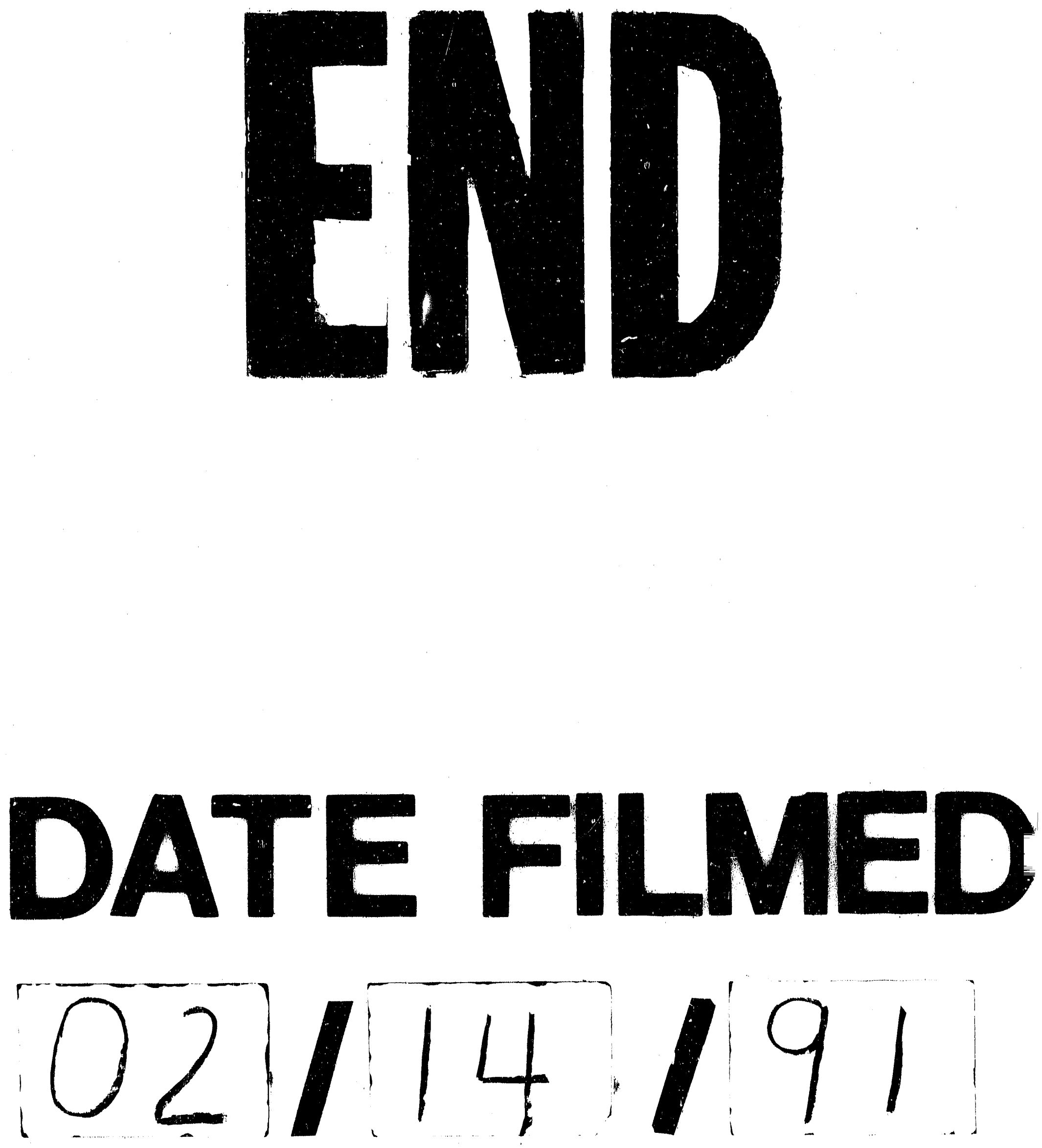
